\title{
SEMIGROUP-THEORETICAL APPROACH TO HIGHER ORDER NONLINEAR EVOLUTION EQUATIONS*
}

\author{
TUJIN KIM ${ }^{\dagger}$, QIANSHUN CHANG $\ddagger$, AND JING XU§
}

\begin{abstract}
We are concerned with application of the semigroup theory to the higher order nonlinear evolution equations. First, we show some necessary conditions for the accretivity of matrices of nonlinear operators in Banach spaces in relation to the underlying phase spaces and domains of operators. Then, we obtain a condition for a matrix of linear operators to generate an analytic semigroup. These results are, finally, applied to Cauchy problems of nonlinear and quasilinear evolution equations of higher order.
\end{abstract}

Key words. Accretivity of operator matrices, analytic semigroup, nonlinear higher order evolution equation, pseudo-hyperbolic system.

AMS subject classifications. 34G20, 35L82, 47D03, 47H20.

1. Introduction. Linear abstract evolution equations have been studied widely (cf. [10], [12], [13], [16], [19], [20], [22], [27], [28], [29]), while nonlinear evolution equations are considered mainly within the scope of the 2-nd order (cf. [1], [3], [4], [7], [15], [18], [23]) and in some papers nonexistence of solution for higher order semilinear evolution equations was studied (cf. [2] and the references therein).

One of the conventional approach to the higher order equations is to reduce them to the first order systems in suitable phase spaces and then to use the operator semigroup theory (cf. the references in [12]). In [5] and [8], a wave equation and a linear parabolic equation of higher order in time, respectively, were reduced to the systems of order one and the analytic semigroup theory was applied for the obtained systems. However, it is generally difficult to find an ideal underlying phase space and the structure of the phase space may be complicated (see preface in [29]). Therefore, for the case of linear equations other techniques are widely used (cf. [29] and the references therein). But, many techniques utilized for linear equations are not applicable for nonlinear equations.

In this paper, we are concerned with the application of the semigroup theory to the higher order nonlinear evolution equations. To this end, we first show some necessary conditions on the phase spaces and operators acting on derivatives of unknown function for accretivity of the matrices of operators in the systems reduced from a simple higher order equations

$$
u^{(n)}+A_{n-1} u^{(n-1)}+\cdots+A_{0} u=f(t), n \geq 2 .
$$

And we get some sufficient conditions for matrices of operators to generate nonlinear one-parameter groups or semigroups. Also, we get a sufficient condition for a matrix of operators to generate an analytic semigroup, which is different from one in [5] and

\footnotetext{
${ }^{*}$ Received January 22, 2011; accepted for publication May 21, 2013. The research of the first author is partially supported by AMSS in Chinese Academy of Sciences, and the third author is partially supported by Youth Foundation of NSFC with Grant No. 11001239.

$\dagger$ Corresponding author. Institute of Mathematics, Academy of Sciences, Pyongyang, DPR Korea (arirangip@star-co.net.kp).

${ }^{\ddagger}$ Institute of Applied Mathematics, AMSS, Chinese Academy of Sciences, Beijing 100190, P. R. China (qschang@amss.ac.cn).

$\S$ School of Statistics and Mathematics, Zhejiang Gongshang University, Hangzhou, China (jingxu@amss.ac.cn).
} 
[8]. The results are applied to Cauchy problems of higher order nonlinear evolution equations. The results of this paper were published in 1990 as preprints of the Institute of Mathematics of the Academy of Sciences in DPR of Korea, but seem to be known to only a few. The main results of this paper are Theorems $2.1 \sim 2.5$ and 4.1.

This paper consists of four sections.

In Section 2 we study the equation (1.1) in a Banach space $E$, where $A_{i}, i=0 \sim$ $n-1$, are usually nonlinear and the derivatives are with respect to $t$. Long ago it was known that when a linear equation of order 2 in $E$ is reduced to a system of order one, for well-posedness the product space $V \times E$ is useful instead of $E \times E$, where the norm of $V$ is stronger than one of $E$ (cf. [13]). On the other hand, the derivatives of function usually are in spaces weaker than one where the function belongs to.

Thus, concerning with $u^{(n)}$ in $E$, we naturally assume

Assumption 1.1. The Banach spaces $V_{i}, i=1 \sim n-1$, and $E$ satisfy the following condition

$$
V_{1} \hookrightarrow V_{2} \hookrightarrow \cdots \hookrightarrow V_{n-1} \hookrightarrow E,
$$

where $\hookrightarrow$ denotes dense and continuous embedding.

And $\mathscr{D}\left(A_{i}\right), i=1 \sim n-1$, and $\mathscr{D}\left(A_{0}\right)$ are dense subsets of Banach spaces $V_{i}$ and $V_{1}$, respectively.

Our first interest is to get necessary conditions for accretivity of the matrices of operators in the system reduced from (1.1). Our result shows that $V_{i}, i=1 \sim n-1$, must be same (Theorem 2.1) and the operators $A_{n-1}$ and $A_{n-2}$ must satisfy some estimations (Theorems $2.3 \sim 2.7$ ).

In Section 3 considering the results in Section 2 and relying on the nonlinear semigroup theory and monotone operator theory, we study three kinds of Cauchy problems for higher order nonlinear evolution equations, which are equations with the perturbation operators of the main part $A_{n-1}$ and $A_{n-2}$. To this end, we obtain some sufficient conditions for the matrices of operators to generate nonlinear semigroups or groups.

In Section 4 the semilinear and quasilinear equations which are not included in the scope of Section 3 are studied. To this end, first, a condition for a matrix of linear operators on Hilbert spaces to generate an analytic semigroup is obtained. Paying attention to role of the operator $A_{n-1}$ and structure of the matrix, and using the space $V^{n-1} \times V^{*}$ as a representation of the dual space of $V^{n}$, we obtain the result without assumptions of self-adjoint property and positive-definiteness of operators (Theorem 4.1). Combining this result with results in [21] and [24], we study Cauchy problems of semilinear and quasilinear evolution equations of higher order. To compare with previous results, we apply our abstract results to the systems of pseudo-hyperbolic partial differential equations. Owing to Theorem 4.1, unlike [25] in study of the systems of pseudo-hyperbolic partial differential equations, symmetry of coefficient matrices is removed out.

We use the following notation.

When $X$ is a space and $X^{*}$ is its dual space, $(\cdot, \cdot)_{X}$ is inner product in $X,\langle\cdot, \cdot\rangle_{X}$ is duality product between $X$ and its dual space $X^{*}$ and $\|\cdot\|_{X}$ is the norm in $X$. Sometimes $\langle\cdot, \cdot\rangle_{i}$ means duality product when $X=V_{i}$, and so is it for norms. For $u_{i}^{1}, u_{i}^{2} \in X$ let $u_{i}=u_{i}^{1}-u_{i}^{2}$. For an operator $A, \mathscr{D}(A)$ is its domain and $\mathscr{R}(A)$ its range. 
2. Necessary conditions for accretivity of operator matrices. Let $V_{i}, i=$ $1, \ldots, n-1$, and $E$ be real Banach spaces and (1.2) hold. Let nonlinear operators $A_{i}, i=1, \ldots, n-1$, in the Banach space $E$ have domains $\mathscr{D}\left(A_{i}\right)$ and $\mathscr{D}\left(A_{0}\right)$ which are dense in $V_{i}$ and $V_{1}$, respectively.

We consider the matrix of operators

$$
\mathbb{A}=\left(\begin{array}{ccccc}
0 & -1 & 0 & \cdots & 0 \\
0 & 0 & -1 & \cdots & 0 \\
& \cdots & & \cdots & \\
A_{0} & A_{1} & A_{2} & \cdots & A_{n-1}
\end{array}\right), \quad \mathscr{D}(\mathbb{A})=\prod_{i=0}^{n-1} \mathscr{D}\left(A_{i}\right)
$$

in the Banach space

$$
\mathbb{X}=V_{1} \times \cdots \times V_{n-1} \times E .
$$

endowed with the norm $\|\cdot\|_{\mathbb{X}}^{2}=\sum_{i=1}^{n-1}\|\cdot\|_{V_{i}}^{2}+\|\cdot\|_{E}^{2}$.

Note that by the introduction of the matrix of operators $\mathbb{A}$ the abstract higher order evolution equation

$$
u^{(n)}+A_{n-1} u^{(n-1)}+\cdots+A_{0} u=f(t)(n \geq 2) \quad \text { in } E
$$

is reduced to the first order system with a new unknown function $\mathbb{U}=$ $\left(u, u_{1}, \ldots, u_{n-1}\right)^{\mathrm{T}}:$

$$
\mathbb{U}^{\prime}+\mathbb{A} \mathbb{U}=F(t) \quad \text { in } \mathbb{X}
$$

where $F(t)=(0, \ldots, 0, f(t))^{\mathrm{T}}$.

Definition 2.1. (cf. 13.2 in [9]) Let $X$ be a real Banach space and $J: X \rightarrow 2^{X^{*}}$ its duality map. The operator $F: \mathscr{D}(F) \subset X \rightarrow X$ is said to be accretive if

$$
\max \left\{\langle F x-F y, j(x-y)\rangle_{X}: j(x-y) \in J(x-y)\right\} \geq 0 \quad \forall x, y \in \mathscr{D}(F) .
$$

If $X^{*}$ is strictly convex, then $J$ is one-value, and so it is said to be duality operator. (cf. Lemma 5.5, ch. 1 in [7])

TheOREm 2.1. Let Assumption 1.1 be valid, $V_{k}, k=1,2, \cdots, n-2$, be reflexive and $V_{k}^{*}$ be strictly convex. If the operator $\mathbb{A}+\omega$ is accretive in $\mathbb{X}$ for some real number $\omega \in \mathcal{R}$, then the spaces $V_{i}, i=1,2, \cdots, n-1$, are the same with equivalent norms, that is, $\mathbb{X}=V^{n-1} \times E$, with a real Banach space $V$.

Proof. Let $\mathbb{U}=\mathbb{U}^{1}-\mathbb{U}^{2}$ for $\mathbb{U}^{1}, \mathbb{U}^{2} \in \mathscr{D}(\mathbb{A})$. Let $V_{n}:=E$. Note that if $j_{k}, k=$ $1,2, \cdots, n$, are duality operators from $V_{k}$ to $V_{k}^{*}$, then the operator $j=\left(j_{1}, \cdots, j_{n}\right)$ defined by $j \mathbb{U}=\left\{j_{1} u_{0}, \cdots, j_{n} u_{n-1}\right\}$ is the duality operator from $\mathbb{X}$ to $\mathbb{X}^{*}$, where $u_{0}=u$. Then, we have

$$
\begin{aligned}
I & \equiv\left\langle(\mathbb{A}+\omega) \mathbb{U}^{1}-(\mathbb{A}+\omega) \mathbb{U}^{2}, j \mathbb{U}\right\rangle_{\mathbb{X}}= \\
& =\sum_{i=0}^{n-2}\left(-\left\langle u_{i+1}, j_{i+1} u_{i}\right\rangle_{i+1}+\omega\left\|u_{i}\right\|_{i+1}^{2}\right)+\sum_{i=0}^{n-1}\left\langle A_{i} u_{i}^{1}-A_{i} u_{i}^{2}, j_{n} u_{n-1}\right\rangle_{n}+\omega\left\|u_{n-1}\right\|_{n}^{2} .
\end{aligned}
$$

Let us prove the equivalence of the norms of $V_{k}, k=1, \ldots, n-1$, by contradiction argument. Assume that for some $k \in\{1, \ldots, n-2\}$ the norms of $V_{k}$ and $V_{k+1}$ are not equivalent. Then, putting $u_{i}^{1}=u_{i}^{2}$ for $i \neq k-1, k$, in (2.3), we have that

$$
I=-\left\langle u_{k}, j_{k} u_{k-1}\right\rangle_{k}+\omega\left\|u_{k-1}\right\|_{k}^{2}+\omega\left\|u_{k}\right\|_{k+1}^{2} .
$$


By the assumption, we have

$$
\forall N \text { (positive integer), } \exists w \in V_{k}:\|w\|_{k} \geq N,\|w\|_{k+1}=1 .
$$

Choose $u_{k}^{1} \in \mathscr{D}\left(A_{k}\right) \subset V_{k}$ and let $\widetilde{u}_{k}^{2}=u_{k}^{1}-w\left(\in V_{k}\right)$. Since $\mathscr{D}\left(A_{k}\right)$ is dense in $V_{k}$, we have

$$
\forall \varepsilon>0, \quad \exists u_{k}^{2} \in \mathscr{D}\left(A_{k}\right):\left\|\widetilde{u}_{k}^{2}-u_{k}^{2}\right\|_{k} \leq \varepsilon,
$$

and, hence, $\left\|u_{k}-w\right\|_{k} \leq \varepsilon$, which together with (2.4) implies

$$
-\left\|u_{k}\right\|_{k} \leq-\|w\|_{k}+\varepsilon \leq-N+\varepsilon
$$

By Assumption 1.1 we get that

$$
\exists r>0:\left\|u_{k}-w\right\|_{k+1} \leq r\left\|u_{k}-w\right\|_{k} \leq r \varepsilon,
$$

which together with (2.4) implies

$$
\left\|u_{k}\right\|_{k+1} \leq\|w\|_{k+1}+r \varepsilon=1+r \varepsilon .
$$

On the other hand,

$$
\exists f \in V_{k}^{*}: \quad\left\langle u_{k}, f\right\rangle_{k}=\left\|u_{k}\right\|_{k}, \quad\|f\|=1 .
$$

The map $j_{k}$ is demicontinuous, d-monotone and coercive (cf. Lemma 5.6, ch. 1 and Remark 1.4, ch. 3 in [7]). Therefore, there exists $j_{k}^{-1} \in\left(V_{k}^{*} \rightarrow V_{k}\right)$, and so

$$
\exists v \in V_{k}: \quad j_{k} v=f,
$$

where $\|v\|_{k}=\|f\|=1$. Choose $u_{k-1}^{1} \in \mathscr{D}\left(A_{k-1}\right) \subset V_{k}$ and let $\widetilde{u}_{k-1}^{2}=u_{k-1}^{1}-v \in V_{k}$. Since $D\left(A_{k-1}\right)$ is dense in $V_{k}$, we can take $u_{k-1}^{2}$ such that $\left\|\widetilde{u}_{k-1}^{2}-u_{k-1}^{2}\right\|_{k}=\| u_{k-1}-$ $v \|_{k}$ is arbitrarily small. Therefore, demicontinuity of $j_{k}$ implies that

$$
\begin{aligned}
\forall \varepsilon>0, \exists u_{k-1}^{1}, u_{k-1}^{2} \in \mathscr{D}\left(A_{k-1}\right): & \left\|u_{k-1}-v\right\|_{k} \leq \varepsilon, \\
& \left|\left\langle u_{k}, j_{k} u_{k-1}\right\rangle_{k}-\left\langle u_{k}, j_{k} v\right\rangle_{k}\right| \leq \varepsilon .
\end{aligned}
$$

Using (2.9) we have

$$
I \leq-\left\langle u_{k}, j_{k} v\right\rangle_{k}+\varepsilon+|\omega|\left(\|v\|_{k}+\varepsilon\right)^{2}+|\omega|\left\|u_{k}\right\|_{k+1}^{2} .
$$

Consequently, $(2.5) \sim(2.10)$ imply

$$
I \leq-N+2 \varepsilon+|\omega|(1+\varepsilon)^{2}+|\omega|(1+r \varepsilon)^{2} .
$$

In (2.11) $r$ is a constant and for the fixed $\varepsilon$ and $\omega$ arbitrarily large integer $N$ can be taken, which is contradictory to accretivity of $\mathbb{A}+\omega$. Therefore, the spaces $V_{k}, k=$ $1 \sim n-1$, are equivalent each other.

REMARK 2.1. Every reflexive Banach space $V$ and its dual $V^{*}$ may be strictly convex by changing the norm of $V$ with a proper equivalent norm. However, since the duality map is variable according to the norms, we assume the strict convexity of $V^{*}$ in Theorem 2.1. 
REMARK 2.2. Accretivity of operator is not a necessary condition to generate a linear one-parameter semigroup and is a part of the sufficient conditions to generate a nonlinear semigroup by Crandall-Liggett (cf. [6]). Thus, in fact, there exists an example of linear semigroup in which under Assumption 1.1 the spaces $V_{i}, i=1 \sim$ $n-1$, are not equivalent (cf. Proposition 1.6 in [8]).

Under consideration of the result above, in Sections 2 and 3 we take the following

Assumption 2.1. Banach spaces $V$ and $E$ are real and $V \hookrightarrow E$. The sets $\mathscr{D}\left(A_{i}\right), i=0 \sim n-1$, are dense in $V$.

THEOREM 2.2. Let the space $V$ be reflexive and $V^{*}$ be strictly convex. Assume the following inequalities:

$$
\begin{gathered}
\left\|A_{n-2} u_{n-2}^{1}-A_{n-2} u_{n-2}^{2}\right\|_{E} \leq K\left\|u_{n-2}\right\|_{V} \quad \forall u_{n-2}^{1}, u_{n-2}^{2} \in \mathscr{D}\left(A_{n-2}\right), \\
\left|\left\langle A_{n-1} u_{n-1}^{1}-A_{n-1} u_{n-1}^{2}, j_{E} u_{n-1}\right\rangle_{E}\right| \leq K\left\|u_{n-1}\right\|_{E}^{2} \forall u_{n-1}^{1}, u_{n-1}^{2} \in \mathscr{D}\left(A_{n-1}\right),
\end{gathered}
$$

where $j_{E}$ is the duality operator from $E$ to $E^{*}$.

Then, the equivalence of the spaces $V$ and $E$, that is, $\mathbb{X}=E^{n}$ is a necessary condition for the existence of a real number $\omega$ such that the operator $\mathbb{A}+\omega$ is accretive in the space $\mathbb{X}=V^{n-1} \times E$.

Proof. Putting $u_{k}^{1}=u_{k}^{2}$ for $k=0,1, \cdots, n-3$ in (2.3) and taking into account the conditions of theorem, we have

$$
I \leq-\left\langle u_{n-1}, j_{V} u_{n-2}\right\rangle_{V}+\omega\left\|u_{n-2}\right\|_{V}^{2}+K_{1}\left\|u_{n-2}\right\|_{E}\left\|u_{n-1}\right\|_{E}+(K+\omega)\left\|u_{n-1}\right\|_{E}^{2},
$$

where $j_{V}$ is the duality operator from $V$ to $V^{*}$.

Thus, as before we come to the asserted conclusion.

TheOREM 2.3. Assume that the space $E$ is reflexive, $E^{*}$ is strictly convex and formula (2.13) holds.

If the operator $\mathbb{A}+\omega$ is accretive in $\mathbb{X}=V^{n-1} \times E$ for some $\omega \in \mathcal{R}$, then we have the followings:

$\mathscr{D}\left(A_{n-1}\right)$,

1) $\left|\left\langle A_{k} u_{k}^{1}-A_{k} u_{k}^{2}, j_{E} u_{k}\right\rangle_{E}\right| \leq M_{k}\left\|u_{k}\right\|_{V}^{2}, k=0,1, \cdots, n-2, \quad \forall u_{k}^{1}, u_{k}^{2} \in \mathscr{D}\left(A_{k}\right) \cap$ where $M_{k}$ are constants;

2) Linear operators among $A_{k}, k=0,1, \cdots, n-3$, are the restrictions of operators belonging to $\mathcal{B} \mathcal{L}(V, E)$;

3) If the set $\mathscr{D}\left(A_{n-1}\right)$ is linear, then

$$
\begin{aligned}
&\left\langle A_{n-2} u_{n-2}^{1}-A_{n-2} u_{n-2}^{2}, j_{E} u_{n-2}\right\rangle \geq a\left\|u_{n-2}\right\|_{V}^{2}+b\left\|u_{n-2}\right\|_{E}^{2} \\
& \forall u_{n-2}^{1}, u_{n-2}^{2} \in \mathscr{D}\left(A_{n-2}\right) \cap \mathscr{D}\left(A_{n-1}\right),
\end{aligned}
$$

where $a>0$ and $b$ is a real number.

Proof of 1). If the dual space of a reflexive Banach space $Y$ is strictly convex, then it is easy to prove that $j_{Y}$ is homogeneous, i.e., $\forall \lambda: j_{Y}(\lambda x)=\lambda j_{Y}(x)$.

Putting $u_{i}^{1}=u_{i}^{2}, i \neq k, n-1$, in (2.3) and using accretivity of $\mathbb{A}+\omega$, we have

$\omega\left\|u_{k}\right\|_{V}^{2}+\left\langle A_{k} u_{k}^{1}-A_{k} u_{k}^{2}, j_{E} u_{n-1}\right\rangle+\left\langle A_{n-1} u_{n-1}^{1}-A_{n-1} u_{n-1}^{2}, j_{E} u_{n-1}\right\rangle+\omega\left\|u_{n-1}\right\|_{E}^{2} \geq 0$. 
Putting $u_{n-1}^{i}=u_{k}^{i}$ in the inequality above, in view of that $V \hookrightarrow E$ which means $\|w\|_{E} \leq r\|w\|_{V} \forall w \in V$, we have

$$
\left\langle A_{k} u_{k}^{1}-A_{k} u_{k}^{2}, j_{E} u_{k}\right\rangle_{E} \geq-\left(K r^{2}+\omega+r^{2}|\omega|\right)\left\|u_{k}\right\|_{V}^{2} .
$$

On the other hand, putting $u_{n-1}^{1}=u_{k}^{2}, u_{n-1}^{2}=u_{k}^{1}$, we have $u_{n-1}=-u_{k}$. Using homogeneity of $j_{E}$, we get

$$
\left\langle A_{k} u_{k}^{1}-A_{k} u_{k}^{2}, j_{E} u_{k}\right\rangle_{E} \leq\left(K r^{2}+\omega+r^{2}|\omega|\right)\left\|u_{k}\right\|_{V}^{2} .
$$

Above two estimates imply the asserted conclusion.

Proof of 2). Suppose that the conclusion is not true. Then, since $\mathscr{D}\left(A_{k}\right)$ is dense in $V$, we have

$$
\forall N, \exists v_{k} \in \mathscr{D}\left(A_{k}\right)\left(\left\|v_{k}\right\|_{V}=1\right):\left\|A_{k} v_{k}\right\|_{E}>N .
$$

Putting $u_{i}^{1}=u_{i}^{2}, i \neq k, n-1$, in (2.3) and using the fact that $A_{k}$ are linear, we get

$$
I=\omega\left\|u_{k}\right\|_{V}+\left\langle A_{k} u_{k}, j_{E} u_{n-1}\right\rangle+\left\langle A_{n-1} u_{n-1}^{1}-A_{n-1} u_{n-1}^{2}, j_{E} u_{n-1}\right\rangle_{E}+\omega\left\|u_{n-1}\right\|_{E}^{2} .
$$

Let $u_{k}=v_{k}$. Then by (2.13) and (2.14),

$$
I \leq \omega-\left\langle-A_{k} u_{k}, j_{E} u_{n-1}\right\rangle_{E}+(K+\omega)\left\|u_{n-1}\right\|_{E}^{2} .
$$

On the other hand, there exists $f \in E^{*}$ such that

$$
\left\langle-A_{k} u_{k}, f\right\rangle_{E}=\left\|-A_{k} u_{k}\right\|_{E},\|f\|=1
$$

As mentioned in the proof of Theorem 2.1, there exists $j_{E}^{-1}$, and so there exists $v \in E$ such that $j_{E} v=f,\|v\|_{E}=1$. Since $\mathscr{D}\left(A_{n-1}\right)$ is dense in $E$ and $j_{E}$ is demicontinuous, using (2.16) and arguing as the proof of Theorem 2.1, we have

$$
\begin{aligned}
\forall \varepsilon>0, \exists u_{n-1}^{1}, u_{n-1}^{2} \in \mathscr{D}\left(A_{n-1}\right): & \left\|u_{n-1}-v\right\|_{E} \leq \varepsilon, \\
& \left\langle-A_{k} u_{k}, j_{E} u_{n-1}\right\rangle \geq\left\|A_{k} u_{k}\right\|_{E}-\varepsilon .
\end{aligned}
$$

From (2.14), (2.15) and (2.17) we have

$$
\begin{aligned}
I & \leq \omega-\left\|A_{k} u_{k}\right\|_{E}+\varepsilon+(K+|\omega|)\left(\|v\|_{E}+\varepsilon\right)^{2} \\
& \leq \omega-N+\varepsilon+(K+|\omega|)(1+\varepsilon)^{2} .
\end{aligned}
$$

This shows contradiction to accretivity of $\mathbb{A}+\omega$, and so we come to the asserted conclusion.

Proof of 3). Let $u_{i}^{1}=u_{i}^{2}, i=0,1, \cdots, n-3$, in (2.3). Taking into account linearity of the set $\mathscr{D}\left(A_{n-1}\right)$, for $\lambda>0$ let $u_{n-1}^{i}=\lambda u_{n-2}^{i} \forall u_{n-2}^{i} \in \mathscr{D}\left(A_{n-2}\right) \cap \mathscr{D}\left(A_{n-1}\right), i=1,2$. Then, the accretivity of $\mathbb{A}+\omega$ implies that

$$
\begin{aligned}
-\left\langle\lambda u_{n-2}, j_{V} u_{n-2}\right\rangle_{V}+\omega\left\|u_{n-2}\right\|_{V}^{2}+\left\langle A_{n-2} u_{n-2}^{1}-A_{n-2} u_{n-2}^{2}, j_{E}\left(\lambda u_{n-2}\right)\right\rangle_{E} \\
+\left\langle A_{n-1}\left(\lambda u_{n-2}^{1}\right)-A_{n-1}\left(\lambda u_{n-2}^{2}\right), j_{E}\left(\lambda u_{n-2}\right)\right\rangle_{E}+\omega\left\|\lambda u_{n-2}\right\|_{E}^{2} \geq 0 .
\end{aligned}
$$

Using homogeneity of $j_{E}$ and (2.13), we have

$$
\left\langle A_{n-2} u_{n-2}^{1}-A_{n-2} u_{n-2}^{2}, j_{E} u_{n-2}\right\rangle_{E} \geq\left(1-\frac{\omega}{\lambda}\right)\left\|u_{n-2}\right\|_{V}^{2}-(K \lambda+\omega \lambda)\left\|u_{n-2}\right\|_{E}^{2} .
$$


Now, taking $\lambda>0$ such that $\left|\frac{\omega}{\lambda}\right|<1$, we come to the asserted conclusion.

ThEOREM 2.4. Let E be a Hilbert space. Assume that

$$
\left\|A_{n-1} u_{n-1}^{1}-A_{n-1} u_{n-2}^{2}\right\|_{V^{*}} \leq K\left\|u_{n-1}\right\|_{V} \forall u_{n-1}^{1}, u_{n-1}^{2} \in \mathscr{D}\left(A_{n-1}\right) .
$$

If $\mathbb{A}+\omega$ is accretive in $\mathbb{X}=V^{n-1} \times E$ with some $\omega \in \mathcal{R}$, then linear operators among $A_{k}, k=0,1, \cdots, n-3$, are the restrictions of bounded linear operators from $V$ to $V^{*}$.

Proof. Suppose that the conclusion is not true. Then, since $\mathscr{D}\left(A_{k}\right)$ is dense in $V$ and $A_{k}$ is linear, it follows that

$$
\begin{aligned}
\forall N, \exists v_{k} \in \mathscr{D}\left(A_{k}\right)\left(\left\|v_{k}\right\|_{V}=1\right), & \exists w \in V\left(\|w\|_{V}=1\right): \\
& \left\langle A_{k} v_{k}, w\right\rangle_{V}=\left(A_{k} v_{k}, w\right)_{E} \geq N .
\end{aligned}
$$

Setting $u_{i}^{1}=u_{i}^{2}, i \neq k, n-1$, in $(2.3)$ we have

$$
I=\omega\left\|u_{k}\right\|_{V}^{2}+\left(A_{k} u_{k}, u_{n-1}\right)_{E}+\left(A_{n-1} u_{n-1}^{1}-A_{n-1} u_{n-1}^{2}, u_{n-1}\right)_{E}+\omega\left\|u_{n-1}\right\|_{E}^{2} .
$$

Putting $u_{k}=-v_{k}$, from this we have that

$$
\begin{aligned}
I \leq \omega\left\|u_{k}\right\|_{V}^{2}-\left(A_{k} v_{k}, u_{n-1}\right)_{E} & \\
& +\left\|A_{n-1} u_{n-1}^{1}-A_{n-1} u_{n-1}^{2}\right\|_{V *} \cdot\left\|u_{n-1}\right\|_{V}+\omega\left\|u_{n-1}\right\|_{E}^{2} \\
& \leq \omega\left\|u_{k}\right\|_{V}^{2}-\left(A_{k} v_{k}, u_{n-1}\right)_{E}+K\left\|u_{n-1}\right\|_{V}^{2}+\omega\left\|u_{n-1}\right\|_{E}^{2} .
\end{aligned}
$$

On the other hand, since $\mathscr{D}\left(A_{n-1}\right)$ is dense in $V$, arguing as the proof of Theorem 2.1 , we have

$$
\begin{aligned}
\forall \varepsilon>0, \exists u_{n-1}^{1}, u_{n-1}^{2} \in \mathscr{D}\left(A_{n-1}\right) & :\left\|w-u_{n-1}\right\|_{V} \leq \varepsilon \\
& -\left(A_{k} v_{k}, u_{n-1}\right)_{E} \leq\left(A_{k} v_{k}, w\right)_{E}+\varepsilon .
\end{aligned}
$$

Then, by (2.18), (2.20) and Assumption 2.1, from (2.19) we have that

$$
\begin{aligned}
I & \leq \omega\left\|u_{k}\right\|_{V}^{2}-\left(A_{k} v_{k}, w\right)_{E}+\varepsilon+K\left\|u_{n-1}\right\|_{V}^{2}+\omega\left\|u_{n-1}\right\|_{E}^{2} \\
& \leq \omega-N+\varepsilon+K(1+\varepsilon)^{2}+|\omega| r^{2}(1+\varepsilon)^{2},
\end{aligned}
$$

where $r$ is the number in the proof of 1 ) of Theorem 2.3. Therefore, we come to a contradiction to accretivity of $\mathbb{A}+\omega$, and so the asserted conclusion is proved.

THEOREM 2.5. Let the space $V$ be reflexive, $V^{*}$ be strictly convex and $A_{n-2} \in$ $\mathcal{L}$ ip $(V, E)$. If the operator $\mathbb{A}+\omega$ is accretive in $\mathbb{X}=V^{n-1} \times E$ with some $\omega \in \mathcal{R}$, then

$$
\left\langle A_{n-1} u_{n-1}^{1}-A_{n-1} u_{n-1}^{2}, j_{E} u_{n-1}\right\rangle \geq a\left\|u_{n-1}\right\|_{V}^{2}+b\left\|u_{n-1}\right\|_{E}^{2} \quad \forall u_{n-1}^{1}, u_{n-1}^{2} \in \mathscr{D}\left(A_{n-1}\right),
$$

where $a>0$ and $b$ is a real number.

Proof. Put $u_{i}^{1}=u_{i}^{2}$ for $i \neq n-1, n-2$. Taking into account $\mathscr{D}\left(A_{n-2}\right)=V$, let $u_{n-2}^{i}=\lambda u_{n-1}^{i}$ for $\lambda>0$ and $u_{n-1}^{1}, u_{n-1}^{2} \in \mathscr{D}\left(A_{n-1}\right)$. Then, by the accretivity of $\mathbb{A}+\omega$ from (2.3) it follows that

$$
\begin{aligned}
& -\left\langle u_{n-1}, j_{V}\left(\lambda u_{n-1}\right)\right\rangle_{V}+\omega\left\|\lambda u_{n-1}\right\|_{V}^{2} \\
& +\left\langle A_{n-2}\left(\lambda u_{n-1}^{1}\right)-A_{n-2}\left(\lambda u_{n-1}^{2}\right), j_{E} u_{n-1}\right\rangle_{E} \\
& +\left\langle A_{n-1} u_{n-1}^{1}-A_{n-1} u_{n-1}^{2}, j_{E} u_{n-1}\right\rangle_{E}+\omega\left\|u_{n-1}\right\|_{E}^{2} \geq 0 .
\end{aligned}
$$


By virtue of homogeneity of $j_{E}$ and the condition of theorem, from (2.21) it follows that for $\varepsilon>0$

$$
\begin{aligned}
\left\langle A_{n-1} u_{n-1}^{1}\right. & \left.-A_{n-1} u_{n-1}^{2}, j_{E} u_{n-1}\right\rangle_{E} \\
& \geq\left(\lambda-\omega \lambda^{2}\right)\left\|u_{n-1}\right\|^{2}-\lambda K\left\|u_{n-1}\right\|_{V} \cdot\left\|u_{n-1}\right\|_{E}-\omega\left\|u_{n-1}\right\|_{E}^{2} \\
& \geq\left(\lambda-\omega \lambda^{2}-\lambda K \frac{\varepsilon}{2}\right)\left\|u_{n-1}\right\|_{V}^{2}-\left(\omega+\lambda K \frac{1}{2 \varepsilon}\right)\left\|u_{n-1}\right\|_{E}^{2},
\end{aligned}
$$

which shows the asserted conclusion.

Similarly, we have the following two Theorems.

THEOREM 2.6. If the operator $\mathbb{A}+\omega$ is accretive in $\mathbb{X}=V^{n-1} \times E$ with some $\omega \in \mathcal{R}$, then the following inequality is valid.

$$
\begin{aligned}
& \forall u_{n-1}^{1}, u_{n-1}^{2} \in \mathscr{D}\left(A_{n-1}\right), \forall u_{n-2}^{1}, u_{n-2}^{2} \in \mathscr{D}\left(A_{n-2}\right), \exists j_{E} u_{n-1}, j_{V} u_{n-2}: \\
& -\left\langle u_{n-1}, j_{V} u_{n-2}\right\rangle_{V}+\omega\left\|u_{n-2}\right\|_{V}^{2}+\left\langle A_{n-2} u_{n-2}^{1}-A_{n-2} u_{n-2}^{2}, j_{E} u_{n-1}\right\rangle_{E} \\
& \quad+\left\langle A_{n-1} u_{n-1}^{1}-A_{n-1} u_{n-1}^{2}, j_{E} u_{n-1}\right\rangle_{E}+\omega\left\|u_{n-1}\right\|_{E}^{2} \geq 0 .
\end{aligned}
$$

If

$$
\left\|A_{k} u_{k}^{1}-A_{k} u_{k}^{2}\right\|_{E} \leq\left\|u_{k}\right\|_{V}, k=0, \cdots, n-3,
$$

then, (2.22) is sufficient for the existence of $\omega_{1} \in \mathcal{R}$ such that $\mathbb{A}+\omega_{1}$ is accretive.

REMARK 2.3. Let $V=H_{0}^{1}(\Omega), E=L_{2}(\Omega)$ and $\mathscr{D}\left(A_{n-1}\right)=\mathscr{D}\left(A_{n-2}\right)=H_{0}^{1}(\Omega) \cap$ $H^{2}(\Omega)$. Define the operators $A_{n-1}$ and $A_{n-2}$, respectively, by

$$
A_{n-1} u_{n-1}=\Sigma_{i} \frac{\partial}{\partial x_{i}} a_{i}\left(x, \cdots, \frac{\partial u_{n-1}}{\partial x_{j}}, \cdots\right)+b\left(x, u_{n-1}, \cdots, \frac{\partial u_{n-1}}{\partial x_{j}}, \cdots\right),
$$

and

$$
A_{n-2} u_{n-2}=\sum_{i} \frac{\partial}{\partial x_{i}} c\left(x, u_{n-2}, \cdots, \frac{\partial u_{n-2}}{\partial x_{j}}, \cdots\right) .
$$

Let the matrix $\left\{a_{i j}\left(x, \cdots, y_{j}, \cdots\right)\right\}$, where $a_{i j}\left(x, \cdots, y_{j}, \cdots\right)=\frac{\partial}{\partial y_{j}} a_{i}\left(x, \cdots, y_{j}, \cdots\right)$, be positive-definite at a.a. $x \in \Omega$ and $a_{i j}\left(\cdot, y_{0}, \cdots, y_{j}, \cdots\right) \in L_{\infty}(\Omega)$ for any $y_{i} \in$ $\mathcal{R} ; b\left(x, y_{0}, \cdots, y_{n}\right), c\left(x, y_{0}, \cdots, y_{n}\right)$ be Lipschitz continuous with respect to $y_{j}, j=$ $0, \cdots, n$. Then, the operators $A_{n-1}$ and $A_{n-2}$ satisfy (2.22).

THEOREM 2.7. For the existence of a real number $\omega \in \mathcal{R}$ such that both $\mathbb{A}+\omega$ and $\mathbb{A}-\omega$ are accretive in $\mathbb{X}=V^{n-1} \times E$ it is necessary that the following inequalities

$$
\begin{aligned}
& \left|-\left\langle u_{n-1}, j_{V} u_{n-2}\right\rangle_{V}+\left\langle A_{n-2} u_{n-2}^{1}-A_{n-2} u_{n-2}^{2}, j_{E} u_{n-1}\right\rangle_{E}\right| \\
& \leq K_{1}\left\|u_{n-2}\right\|_{V}^{2}+K_{2}\left\|u_{n-1}\right\|_{E}^{2}, \\
& \left|\left\langle A_{n-1} u_{n-1}^{1}-A_{n-1} u_{n-1}^{2}, j_{E} u_{n-1}\right\rangle_{E}\right| \leq K_{3}\left\|u_{n-1}\right\|_{E}^{2} .
\end{aligned}
$$

are valid.

If (2.23) is valid, then (2.24) is sufficient for the existence of such an $\omega$. 
REMARK 2.4. Let $a_{i j}(x)=a_{j i}(x), a_{i j}(x) \in W_{\infty}^{1}(\Omega)$ and the matrix $\left\{a_{i j}(x)\right\}$ be positive-definite at a.a. $x \in \Omega$. Let $V=H_{0}^{1}(\Omega)$, where an inner product is one by $(u, v)=\sum_{i j} \int_{\Omega} a_{i j}(x) \frac{\partial u}{\partial x_{i}} \frac{\partial v}{x_{j}} d x, E=L_{2}(\Omega), \mathscr{D}\left(A_{n-1}\right)=H_{0}^{1}(\Omega)$ and $\mathscr{D}\left(A_{n-2}\right)=$ $H_{0}^{1}(\Omega) \cap H^{2}(\Omega)$. Define the operators $A_{n-1}$ and $A_{n-2}$, respectively, by

$$
A_{n-1} u_{n-1}=\sum_{i} a_{i}(x) \frac{\partial u_{n-2}}{\partial x_{i}}+b\left(x, u_{n-1}\right)
$$

and

$$
A_{n-2} u_{n-2}=\sum_{i j} \frac{\partial}{\partial x_{i}}\left(a_{i j}(x) \frac{\partial u_{n-2}}{\partial x_{j}}\right)+c\left(x, u_{n-2}, \cdots, \frac{\partial u_{n-2}}{\partial x_{j}}, \cdots\right),
$$

where $b\left(x, y_{0}\right), c\left(x, y_{0}, \cdots, y_{n}\right)$ are Lipschitz continuous with respect to $y_{j}, j=$ $0, \cdots, n$. Then, the operators $A_{n-1}$ and $A_{n-2}$ satisfy (2.24).

\section{Cauchy problems of some nonlinear equations.}

Definition 3.1. If $u \in C^{n-2}\left(\left[T_{0}, T\right] ; V\right), u^{(n-1)} \in C\left(\left[T_{0}, T\right] ; E\right)$ and

$$
u^{(n)}+A\left(u, \cdots, u^{(n-1)}\right)=f(t) \quad \text { for a.a. } t \in\left[T_{0}, T\right] \text { in } E,
$$

then, $u$ is called a strong solution to $(*)$ on $\left[T_{0}, T\right]$.

The results above show that accretivity of $\mathbb{A}+\omega$ depends mainly on characters of the operators acting on $u^{(n-1)}$ and $u^{(n-2)}$. Therefore, we will consider three kinds of equations in view of the operators acting on $u^{(n-1)}$ and $u^{(n-2)}$.

First, we study a Cauchy problem

$$
\begin{aligned}
& u^{(n)}+A_{n-1} u^{(n-1)}+A_{n-2} u^{(n-2)}+A\left(u, \cdots, u^{(n-1)}\right)=f(t), \\
& u(0)=u_{0}, u^{\prime}(0)=u_{0}^{\prime}, \cdots, u^{(n-1)}(0)=u_{0}^{(n-1)} .
\end{aligned}
$$

LEMma 3.1. Let $E$ be a real Hilbert space and $V$ be a real reflexive Banach space. Suppose that

1) $A \in \mathcal{L}$ ip $\left(V^{n-1} \times E, E\right)$;

2) $A_{n-1} \in \mathcal{L} i p(V, E)$ and

$$
\left|\left(A_{n-1} u_{n-1}^{1}-A_{n-1} u_{n-1}^{2}, u_{n-1}\right)_{E}\right| \leq K\left\|u_{n-1}\right\|_{E}^{2} ;
$$

3) $A_{n-2}$ is a restriction on $E$ of a radially continuous (cf. Definition 1.1, ch. 3 in [7]) operator $\widetilde{A}_{n-2}$ from $V$ to $V^{*}$ such that

$$
\left\langle\widetilde{A}_{n-2} u_{n-2}^{1}-\widetilde{A}_{n-2} u_{n-2}^{2}, u_{n-2}\right\rangle_{V} \geq a\left\|u_{n-2}\right\|_{V}^{2}+b\left\|u_{n-2}\right\|_{E}^{2},
$$

where $a>0$ and $b$ is a real number.

Then,

$$
\exists \lambda_{0}>0, \forall \lambda\left(0<|\lambda| \leq \lambda_{0}\right): \mathscr{R}(I+\lambda \mathbb{A})=\mathbb{X},
$$

where $I$ is the unit operator on $\mathbb{X}$ and

$$
\mathbb{A} \mathbb{U}=\left(\begin{array}{c}
-u_{1} \\
-u_{2} \\
\vdots \\
A_{n-1} u_{n-1}+A_{n-2} u_{n-2}+A\left(u, \cdots, u_{n-1}\right)
\end{array}\right), \mathscr{D}(\mathbb{A})=\left(\begin{array}{c}
V \\
\vdots \\
\mathscr{D}\left(A_{n-2}\right) \\
V
\end{array}\right) \text {. }
$$


Proof. The asserted conclusion is equivalent to the existence of a solution to the following problem

$$
(\lambda+\mathbb{A}) \mathbb{U}=F, F=\left(\begin{array}{c}
f_{0} \\
\vdots \\
f_{n-1}
\end{array}\right) \in \mathbb{X} \quad \forall \lambda\left(|\lambda| \geq \tilde{\lambda}_{0}\right),
$$

where $\widetilde{\lambda}_{0}$ is a constant. Equation (3.5) is equivalent to the following system

$$
\begin{aligned}
& u_{n-1}=\lambda u_{n-2}-f_{n-2}, \\
& u_{n-3}=\frac{f_{n-3}+u_{n-2}}{\lambda}, \\
& \text { ……, } \\
& u_{i}=\sum_{k=i}^{n-3} \frac{f_{k}}{\lambda^{k-i+1}}+\frac{u_{n-2}}{\lambda^{n-2-i}}, \\
& \text {........., } \\
& u=\sum_{k=0}^{n-3} \frac{f_{k}}{\lambda^{k+1}}+\frac{u_{n-2}}{\lambda^{n-2}}, \\
& A\left(\sum_{k=0}^{n-3} \frac{f_{k}}{\lambda^{k+1}}+\frac{u_{n-2}}{\lambda^{n-2}}, \cdots, \sum_{k=i}^{n-3} \frac{f_{k}}{\lambda^{k-i+1}}+\frac{u_{n-2}}{\lambda^{n-2-i}}, \cdots, u_{n-2}, \lambda u_{n-2}-f_{n-2}\right) \\
& +A_{n-2} u_{n-2}+A_{n-1}\left(\lambda u_{n-2}-f_{n-2}\right)+\lambda^{2} u_{n-2}-\lambda f_{n-2}=f_{n-1} .
\end{aligned}
$$

If there exists a solution $u_{n-2}$ to the last equation of (3.6), substituting it into other we have a solution to (3.5).

Let us prove the existence of a solution to the last equation of (3.6). Replacing $A_{n-2}$ with $\widetilde{A}_{n-2}$ and denoting the left-hand side of the last one in (3.6) by $\widetilde{A}_{\lambda}$, we have the equation

$$
\widetilde{A}_{\lambda} u=f_{n-1} .
$$

Since an arbitrarily large $\bar{\lambda}_{0}$ can be taken, we can assume that $|\lambda|>1$ without loss of generality. Using the conditions of theorem and the fact that $\langle v, u\rangle_{V}=(v, u)_{E}$ for $u \in V, v \in E$, we have

$$
\begin{aligned}
& \left\langle\widetilde{A}_{\lambda} u^{1}-\widetilde{A}_{\lambda} u^{2}, u\right\rangle \\
& \geq-M \sum_{i=0}^{n-3} \frac{\|u\|_{V} \cdot\|u\|_{E}}{|\lambda|^{n-2-i}}-M\|u\|_{V} \cdot\|u\|_{E}-M|\lambda|\|u\|_{E}^{2}+\left\langle\widetilde{A}_{n-2} u^{1}-\widetilde{A}_{n-2} u^{2}, u\right\rangle_{V} \\
& \quad+\frac{1}{\lambda}\left(A_{n-1}\left(\lambda u^{1}-f_{n-2}\right)-A_{n-1}\left(\lambda u^{2}-f_{n-2}\right), \lambda u\right)+\lambda^{2}\|u\|_{E}^{2} \\
& \geq-\frac{M}{|\lambda|-1}\|u\|_{V}\|u\|_{E}-M\|u\|_{V} \cdot\|u\|_{E}-M|\lambda|\|u\|_{E}^{2} \\
& \quad+a\|u\|_{V}^{2}+b\|u\|_{E}^{2}-\frac{K}{|\lambda|}\|\lambda u\|_{E}^{2}+\lambda^{2}\|u\|_{E}^{2} \\
& \geq\left(a-\frac{M}{2(|\lambda|-1)}-\frac{\varepsilon M}{2}\right)\|u\|_{V}^{2}+\left(\lambda^{2}-M|\lambda|-K|\lambda|+b-\frac{M}{2(|\lambda|-1)}-\frac{M}{2 \varepsilon}\right)\|u\|_{E}^{2} \\
& \quad \forall u^{1}, u^{2} \in V .
\end{aligned}
$$


Taking $\varepsilon=\frac{a}{2 M}$, we can see that $\widetilde{A}_{\lambda}$ is strongly monotone for all $\lambda\left(|\lambda|>\bar{\lambda}_{0}\right)$ provided $\bar{\lambda}_{0}$ is large enough. On the other hand, by the conditions of theorem the operator $\widetilde{A}_{\lambda} \in\left(V \rightarrow V^{*}\right)$ is radially continuous. Hence, there exists a solution $\bar{u} \in V$ to equation (3.7). (cf. Theorem 2.1 of ch. 3 in [7]) From (3.7) we get

$$
\begin{gathered}
\widetilde{A}_{n-2} \bar{u}=f_{n-1}-A\left(\cdots, \sum_{k=i}^{n-3}\left(\frac{f_{k}}{\lambda^{k-i+1}}+\frac{\bar{u}}{\lambda^{n-2-i}}\right), \cdots\right) \\
-A_{n-1}\left(\lambda \bar{u}-f_{n-2}\right)-\lambda^{2} \bar{u}+\lambda f_{n-2} .
\end{gathered}
$$

Since $f_{0}, \cdots, f_{n-2} \in V, f_{n-1} \in E$ and $\bar{u} \in V$, the right hand side of (3.8) is an element of $E$. Thus, $\widetilde{A}_{n-2} \bar{u} \in E$, which shows that $\bar{u} \in \mathscr{D}\left(A_{n-2}\right)$. Thus, we proved the existence of a solution to the last equation of (3.6).

Lemma 3.2. Suppose that in a Banach space for some $\omega \in R$ both operators $B+\omega$ and $-B+\omega$ are accretive and both $B$ and $-B$ generate, respectively, nonlinear semigroups $T_{+}(t)$ and $T_{-}(t), t \in[0, \infty)$, on $\overline{\mathscr{D}(B)}$. If $T_{+}(t) x_{0}$ and $T_{-}(t) x_{0}$ for $x_{0} \in$ $\mathscr{D}(B)$ are, respectively, unique strong solutions to the equations $\dot{x}+B x=0$ and $\dot{x}-B x=0, t \in[0, \infty)$, then the operator $B$ generates one-parameter nonlinear group on $\overline{\mathscr{D}(B)} U(t), t \in(-\infty, \infty)$, and

$$
U(t+r)=T_{\operatorname{sign}(\mathrm{t})}(|t|) \cdot T_{\operatorname{sign}(\mathrm{r})}(|r|) \forall t \in(-\infty, \infty) .
$$

Proof. Under consideration of the uniqueness of a strong solution to $\dot{x}+B x=$ $0, t \in(-\infty, \infty)$, we can prove it.

TheOREM 3.3. Let $V$ and $E$ be real Hilbert spaces such that $V \hookrightarrow E$. Assume that the conditions 1) and 2) in Lemma 3.1 hold.

If $A_{n-2}$ is the restriction on $E$ of a self-adjoint and strongly monotone operator $\widetilde{A}_{n-2} \in \mathcal{B L}\left(V, V^{*}\right)$, then the operator $\mathbb{A}$ in (3.4) generates a nonlinear group on $\mathbb{X}=V^{n-1} \times E$. And so, when $\mathbb{U}_{0} \equiv\left(u_{0}, u_{0}^{\prime}, \cdots, u_{0}^{(n-1)}\right) \in \mathscr{D}(\mathbb{A})$ and $f \in \mathcal{B V}([-T, T] ; E), T>0$ (the space of functions with bounded variation), there exists a unique strong solution to (3.1) on $[-T, T]$.

Proof. A new inner product in $V$ can be introduced by $\left\langle\widetilde{A}_{n-2} u, v\right\rangle_{V}$. Denote by $\widetilde{V}$ the new Hilbert space with this inner product. Then,

$$
-\left(u_{n-1}, u_{n-2}\right)_{\tilde{V}}+\left(A_{n-2} u_{n-2}, u_{n-1}\right)_{E}=0 \quad \forall u_{n-2}^{1}, u_{n-2}^{2} \in \mathscr{D}\left(A_{n-2}\right) .
$$

Let us prove that the operator $\mathbb{A}+\omega$ for an $\omega \in \mathcal{R}$ is accretive in the space $\widetilde{\mathbb{X}}=\widetilde{V}^{n-1} \times E$. Taking into account $(3.9)$ and the fact that $A \in \mathcal{L} i p\left(\widetilde{V}^{n-1} \times E, E\right)$, we have that

$$
\begin{aligned}
I \equiv & \left(\mathbb{A U}^{1}-\mathbb{A U}^{2}, \mathbb{U}\right)_{\widetilde{\mathbb{X}}}= \\
=- & \sum_{i=0}^{n-2}\left(u_{i+1}, u_{i}\right)_{\widetilde{V}}+\left(A_{n-1} u_{n-1}^{1}-A_{n-1} u_{n-1}^{2}, u_{n-1}\right)_{E}+\left(A_{n-2} u_{n-2}, u_{n-1}\right)_{E} \\
& \quad+\left(A\left(u^{1}, u_{1}^{1}, \cdots, u_{n-2}^{1}, u_{n-1}^{1}\right)-A\left(u^{2}, u_{1}^{2}, \cdots, u_{n-2}^{2}, u_{n-1}^{2}\right), u_{n-1}\right)_{E} \\
& \geq-\sum_{i=0}^{n-3}\left(\frac{\left\|u_{i+1}\right\|_{\widetilde{V}}^{2}}{2}+\frac{\left\|u_{i}\right\|_{\widetilde{V}}^{2}}{2}\right)-\frac{M^{2}}{2} \sum_{i=0}^{n-2}\left\|u_{i}\right\|_{\widetilde{V}}^{2}-\frac{1}{2}\left\|u_{n-1}\right\|_{E}^{2}-\frac{M^{2}}{2}\left\|u_{n-1}\right\|_{E}^{2} \\
\geq- & \frac{M^{2}+2}{2} \sum_{i=0}^{n-3}\left\|u_{i}\right\|_{\widetilde{V}}^{2}-\frac{M^{2}+1}{2}\left\|u_{n-2}\right\|_{\widetilde{V}}^{2}-\frac{M^{2}+1}{2}\left\|u_{n-1}\right\|_{E}^{2},
\end{aligned}
$$


where as before $u_{0}=u$. Thus, putting $\omega=\frac{M^{2}+2}{2}$, we see that $\mathbb{A}+\omega$ is accretive in the space $\widetilde{\mathbb{X}}=\widetilde{V}^{n-1} \times E$. In the same way we can prove that $-\mathbb{A}+\omega$ is also accretive in $\widetilde{\mathbb{X}}$.

By Lemma 3.1, we obtain that $\mathscr{R}(I+\lambda \mathbb{A})=\widetilde{\mathbb{X}}, \mathscr{R}(I-\lambda \mathbb{A})=\widetilde{\mathbb{X}}$ for any $\lambda$ small enough. We can see that $\mathscr{D}(\mathbb{A})=\mathscr{D}(-\mathbb{A})$ is dense in $\mathbb{X}$. Thus, $\mathbb{A}$ and $-\mathbb{A}$ generate, respectively, nonlinear semigroups on $\mathbb{X}$, and so by Lemma 3.2 the operator $\mathbb{A}$ generates a nonlinear group. Existence of a unique strong solution to (3.1) follows by the semigroup theory (cf. [14]).

Second, we study the following Cauchy problem

$$
\begin{aligned}
& u^{(n)}+A_{n-1} u^{(n-1)}+A\left(u, \cdots, u^{(n-1)}\right)=f(t), \\
& u(0)=u_{0}, u^{\prime}(0)=u_{0}^{\prime}, \cdots, u^{(n-1)}(0)=u_{0}^{(n-1)} .
\end{aligned}
$$

For (3.10) Definition 3.1 is used with $V \equiv E$.

Theorem 3.4. Suppose that $A \in \mathcal{L} i p\left(E^{n}, E\right), \mathscr{D}\left(A_{n-1}\right)$ is dense in $E$, formula (2.13) holds and

$$
\exists \lambda_{0}>0, \forall \lambda\left(|\lambda| \geq \lambda_{0}\right): \mathscr{R}\left(\lambda+A_{n-1}\right)=E .
$$

Then, both $\mathbb{A}$ and $-\mathbb{A}$ generate nonlinear semigroups on $\mathbb{X}=E^{n}$, where

$$
\mathscr{D}(\mathbb{A})=\left(\begin{array}{c}
E \\
\vdots \\
E \\
\mathscr{D}\left(A_{n-1}\right)
\end{array}\right), \mathbb{A} \mathbb{U}=\left(\begin{array}{c}
-u_{1} \\
-u_{2} \\
\vdots \\
A_{n-1} u_{n-1}+A\left(u, \cdots, u_{n-1}\right)
\end{array}\right) .
$$

If $E$ is reflexive, then $\mathbb{A}$ generates a nonlinear group. If $E$ is reflexive, $\mathbb{U}_{0} \in \mathscr{D}(\mathbb{A})$ and $f(t) \in \mathcal{B V}([-T, T] ; E)$, then there exists a unique strong solution to (3.10) on $[-T, T]$.

The proof is similar to the case of Theorem 3.3 and is omitted.

REMARK 3.1. An example of linear partial differential operator satisfying the conditions (2.13) and (3.11) is shown in 3.5 in [26].

Finally, we consider the following Cauchy problem

$$
\begin{aligned}
& u^{(n)}+A\left(u, u^{\prime}, \cdots, u^{(n-1)}\right)=f(t), \\
& u(0)=u_{0}, u^{\prime}(0)=u_{0}^{\prime}, \cdots, u^{(n-1)}(0)=u_{0}^{(n-1)}, \mathbb{U}_{0} \equiv\left(u_{0}, u_{0}^{\prime}, \cdots, u_{0}^{(n-1)}\right) .
\end{aligned}
$$

TheOREm 3.5. Let $V$ and $E$ be real Hilbert spaces such that $V \hookrightarrow E$. Suppose that $A \in \mathcal{L} i p\left(V^{n}, V^{*}\right)$ and

$$
\begin{aligned}
\left\langle A\left(u, u_{1}, \cdots, u_{n-2}, u_{n-1}^{1}\right)-A\left(u, u_{1}, \cdots, u_{n-2}, u_{n-1}^{2}\right), u_{n-1}\right\rangle_{V} & \\
& \geq a\left\|u_{n-1}\right\|_{V}^{2}+b\left\|u_{n-1}\right\|_{E}^{2} \quad \forall u, u_{1}, \cdots, u_{n-2}, u_{n-1}^{1}, u_{n-1}^{2} \in V,
\end{aligned}
$$

where $a>0$ and $b$ is real number. 
Then, the restriction of $\mathbb{A}$ to $\mathbb{X}=V^{n-1} \times E$ generates a nonlinear semigroup, where

$$
\mathbb{A} \mathbb{U}=\left(\begin{array}{c}
-u_{1} \\
\vdots \\
-u_{n-1} \\
A\left(u, u_{1}, \cdots, u_{n-1}\right)
\end{array}\right)
$$

and so there exists a unique strong solution to $(3.12)$ on $[0, T]$ for $\mathbb{U}_{0} \in \mathscr{D}(\mathbb{A})$ and $f(t) \in \mathcal{B V}([0, T], E)$. If $\mathbb{U}_{0} \in \mathbb{X}$ and $f(t) \in L^{2}\left(0, T ; V^{*}\right)$, then there exists a unique solution $u \in C^{n-2}([0, T] ; V)$ such that $u^{(n-1)} \in L^{2}(0, T ; V) \cap C([0, T] ; E)$ and $u^{(n)} \in$ $L_{2}\left(0, T ; V^{*}\right)$.

Proof. Let $\mathbb{Y}=V^{n}$. According to Riesz theorem, let us identify every component of $\left(V^{*}\right)^{n}$ with the exception of the last one, with $V$. Then, we can regard $\mathbb{Y}^{*}=$ $V^{n-1} \times V^{*}$ as a representation of the dual space of $\mathbb{Y}$, and $\mathbb{A}$ is an operator from $\mathscr{D}(\mathbb{A})=\mathbb{Y}$ to $\mathbb{Y}^{*}$. If the space $\mathbb{X}=V^{n-1} \times E$ is identified with $\mathbb{X}^{*}$, then we get

$$
\mathbb{Y} \hookrightarrow \mathbb{X} \hookrightarrow \mathbb{Y}^{*}
$$

Thus, we have

$$
\begin{aligned}
& \forall \mathbb{U}^{1}, \mathbb{U}^{2} \in \mathbb{Y}:\left\langle A \mathbb{U}^{1}-\mathbb{A} \mathbb{U}^{2}, \mathbb{U}\right\rangle_{\mathbb{Y}}= \\
& \begin{aligned}
n-\sum_{i=0}^{n-2}\left(u_{i+1}, u_{i}\right)_{V}+\left\langle A\left(u^{1}, u_{1}^{1}, \cdots, u_{n-2}^{1}, u_{n-1}^{1}\right)-A\left(u^{1}, u_{1}^{1}, \cdots, u_{n-2}^{1}, u_{n-1}^{2}\right), u_{n-1}\right\rangle_{V} \\
\quad+\left\langle A\left(u^{1}, u_{1}^{1}, \cdots, u_{n-2}^{1}, u_{n-1}^{2}\right)-A\left(u^{2}, u_{1}^{2}, \cdots, u_{n-2}^{2}, u_{n-1}^{2}\right), u_{n-1}\right\rangle_{V} \\
\geq-\sum_{i=0}^{n-3}\left(\frac{\left\|u_{i+1}\right\|_{V}^{2}}{2}+\frac{\left\|u_{i}\right\|_{V}^{2}}{2}\right)-\left\|u_{n-1}\right\|_{V} \cdot\left\|u_{n-2}\right\|_{V} \\
\quad+a\left\|u_{n-1}\right\|_{V}^{2}+b\left\|u_{n-1}\right\|_{E}^{2}-M \sum_{i=0}^{n-2}\left\|u_{i}\right\|_{V} \cdot\left\|u_{n-1}\right\|_{V} \\
\geq-\sum_{i=0}^{n-3}\left(\frac{\left\|u_{i+1}\right\|_{V}^{2}}{2}+\frac{\left\|u_{i}\right\|_{V}^{2}}{2}\right)-\left\|u_{n-1}\right\|_{V} \cdot\left\|u_{n-2}\right\|_{V} \\
\quad-M \sum_{i=0}^{n-2}\left\|u_{i}\right\|_{V} \cdot\left\|u_{n-1}\right\|_{V}+a\left\|u_{n-1}\right\|_{V}^{2}+b\left\|u_{n-1}\right\|_{E}^{2} \\
\geq\left[a-\frac{\varepsilon}{2}(M(n-1)+1)\right]\left\|u_{n-1}\right\|_{V}^{2}-\left(\frac{M+1}{2 \varepsilon}+1\right)\left\|u_{n-2}\right\|_{V}^{2} \\
-\left(\frac{M}{2 \varepsilon}+1\right) \sum_{i=0}^{n-3}\left\|u_{i}\right\|_{V}^{2}+b\left\|u_{n-1}\right\|_{E}^{2},
\end{aligned}
\end{aligned}
$$

where $\varepsilon>0, \mathrm{M}$ is the Lipschitz constant of $A, \mathbb{U}=\mathbb{U}^{1}-\mathbb{U}^{2}$ and $u_{0}=u$.

Let $\varepsilon=\frac{a}{M(n-1)+1}, \omega=\max \left\{\frac{M+1}{2 \varepsilon}+1,-b\right\}+\delta, \delta>0$. Then $\mathbb{A}+\omega I$ is a strongly monotone Lipschitz operator, where $I$ is embedding operator from $\mathbb{Y}$ into $\mathbb{Y}^{*}$ by (3.13). Therefore, by the semigroup theory (cf. [14]), the operator $\mathbb{A}$ generates a nonlinear semigroup on $\mathbb{X}$. Also, by Theorem 1.3, ch. 6 in [7], we come to the last conclusion. $\square$

Relying on Theorem 1.1 of ch. 6 in [7], in the same way we have 
Theorem 3.6. Let $A$ be a Volterra operator such that $A \in \mathcal{L}$ ip $\left(L_{2}(0, T ; V)^{n} \rightarrow\right.$ $\left.L_{2}\left(0, T ; V^{*}\right)\right)$, and

$$
\begin{aligned}
&\left\langle A\left(u, \cdots, u_{n-2}, u_{n-1}^{1}\right)-\right.\left.A\left(u, \cdots, u_{n-2}, u_{n-1}^{2}\right), u_{n-1}\right\rangle_{L_{2}(0, T ; V)} \\
& \geq a\left\|u_{n-1}\right\|_{L_{2}(0, T ; V)}^{2}+b\left\|u_{n-1}\right\|_{L_{2}(0, T ; E)}^{2} \\
& \forall u, \cdots, u_{n-2}, u_{n-1}^{1}, u_{n-1}^{2} \in L_{2}(0, T ; V),
\end{aligned}
$$

where $a>0$ and $b$ is a real number. Also, let $\mathbb{U}_{0} \in V^{n-1} \times E$ and $f \in L_{2}\left(0, T ; V^{*}\right)$.

Then, there exists a unique solution to (3.12) such that $u \in$ $C^{n-2}([0, T] ; V), u^{(n-1)} \in C([0, T] ; H) \cap L_{2}(0, T ; V)$ and $u^{(n)} \in L_{2}\left(0, T ; V^{*}\right)$.

REMARK 3.2. Theorems 3.3 and 3.4 have applications in semilinear hyperbolic differential equations, and Theorems 3.5 and 3.6 do it in pseudo-hyperbolic differential equations. But here we omit the concrete examples for application .

The assertion of generation of nonlinear group in Theorems 3.3 and 3.4 may be obtained by perturbation of a generator of linear operator semigroup with a Lipschitz operator.

Theorem 3.5 can not be applied to PDE with coefficients variable in time $t$, but Theorem 3.6 is useful for such cases.

4. Analytic semigroup and semilinear and quasilinear equations. In this section, first, we get a sufficient condition for a matrix of operators to generate an analytic semigroup.

Theorem 4.1. Let $V$ and $E$ be Hilbert spaces such that $V \hookrightarrow E$. Suppose that $A_{i} \in \mathcal{B L}\left(V, V^{*}\right), i=0,1, \cdots, n-1$, and there exists a constant $m>0$ and a real number $k$ such that

$$
\operatorname{Re}\left\langle A_{n-1} u, u\right\rangle_{V} \geq m\|u\|_{V}^{2}+k\|u\|_{E}^{2} \quad \forall u \in V,
$$

where $V^{*}$ is the space of all antilinear continuous functionals on $V$.

Then, the operator $\mathbb{A}$ generates an analytic semigroups on $V^{n-1} \times V^{*}$ and so does the restriction of $\mathbb{A}$ on $V^{n-1} \times E$, where

$$
\mathbb{A}=\left(\begin{array}{ccccc}
0 & 1 & 0 & \cdots & 0 \\
0 & 0 & 1 & \cdots & 0 \\
& \cdots & & \cdots & \\
-A_{0} & -A_{1} & -A_{2} & \cdots & -A_{n-1}
\end{array}\right)
$$

Proof. Let $\mathbb{Y}=V^{n}, \mathbb{X}=V^{n-1} \times E$. Then, as the proof of Theorem 3.5 we can write $\mathbb{Y}^{*}=V^{n-1} \times V^{*}($ cf. 2.2 in $[26])$ and $\mathbb{A}$ is an operator from $\mathscr{D}(\mathbb{A})=\mathbb{Y}$ to $\mathbb{Y}^{*}$. Define a functional on $\mathbb{Y} \times \mathbb{Y}$ by

$$
a(\mathbb{U}, \mathbb{V}) \equiv\langle(-\mathbb{A}+\omega I) \mathbb{U}, \mathbb{V}\rangle_{Y} \text { for } \mathbb{U}, \mathbb{V} \in \mathbb{Y}
$$


where $\omega$ is a real number determined later. Let $\varepsilon>0$ and $M=$ $\max _{0 \leq i \leq n-2}\left\{\left\|A_{i}\right\|_{V \rightarrow V^{*}}\right\}$. Then, we have

$$
\begin{aligned}
& \operatorname{Re} a(\mathbb{U}, \mathbb{U}) \\
& =\operatorname{Re}\left(-\sum_{i=0}^{n-2}\left(u_{i+1}, u_{i}\right)_{V}+\sum_{k=0}^{n-1}\left\langle A_{k} u_{k}, u_{n-1}\right\rangle_{V}\right)+\omega \sum_{i=0}^{n-2}\left\|u_{i}\right\|_{V}^{2}+\omega\left\|u_{n-1}\right\|_{E}^{2} \\
& \geq-\sum_{i=0}^{n-3}\left(\frac{\left\|u_{i+1}\right\|_{V}^{2}}{2}+\frac{\left\|u_{i}\right\|_{V}^{2}}{2}\right)-\left\|u_{n-1}\right\|_{V} \cdot\left\|u_{n-2}\right\|_{V}+m\left\|u_{n-1}\right\|_{V}^{2}+k\left\|u_{n-1}\right\|_{E}^{2} \\
& \quad-M \sum_{i=0}^{n-2}\left\|u_{i}\right\|_{V}\left\|u_{n-1}\right\|_{V}+\omega \sum_{i=0}^{n-2}\left\|u_{i}\right\|_{V}^{2}+\omega\left\|u_{n-1}\right\|_{E}^{2} \\
& \quad \sum_{i=0}^{n-3}\left\|u_{i}\right\|_{V}^{2}+\frac{\left\|u_{0}\right\|_{V}^{2}}{2}-\frac{\left\|u_{n-2}\right\|_{V}^{2}}{2}-\frac{1}{2 \varepsilon}\left\|u_{n-2}\right\|_{V}^{2}-\frac{\varepsilon}{2}\left\|u_{n-1}\right\|_{V}^{2}-M \sum_{i=0}^{n-2} \frac{1}{2 \varepsilon}\left\|u_{i}\right\|_{V}^{2} \\
& \quad-\frac{M \varepsilon}{2}(n-1)\left\|u_{n-1}\right\|_{V}^{2}+m\left\|u_{n-1}\right\|_{V}^{2}+k\left\|u_{n-1}\right\|_{E}^{2}+\omega \sum_{i=0}^{n-2}\left\|u_{i}\right\|_{V}^{2}+\omega\left\|u_{n-1}\right\|_{E}^{2} \\
& \geq\left[m-\frac{\varepsilon}{2}(M(n-1)+1)\right]\left\|u_{n-1}\right\|_{V}^{2}-\left(\frac{M}{2 \varepsilon}+1\right) \sum_{i=0}^{n-3}\left\|u_{i}\right\|_{V}^{2} \\
& \quad-\left(\frac{M+1}{2 \varepsilon}+\frac{1}{2}\right)\left\|u_{n-2}\right\|_{V}^{2}+k\left\|u_{n-1}\right\|_{E}^{2}+\omega \sum_{i=0}^{n}\left\|u_{i}\right\|_{V}^{2}+\omega\left\|_{n-1}\right\|_{E}^{2} \cdot
\end{aligned}
$$

Now, putting

$$
\varepsilon=\frac{m}{M(n-1)+1}, \quad \omega=\max \left\{\frac{M+1}{2 \varepsilon}+1,-k\right\}+\delta, \quad \delta>0
$$

we have

$$
R e\langle(-\mathbb{A}+\omega I) \mathbb{U}, \mathbb{U}\rangle_{\mathbb{Y}} \geq \frac{m}{2}\left\|u_{n-1}\right\|_{V}^{2}+\delta \sum_{i=0}^{n-2}\left\|u_{i}\right\|_{V}^{2} \geq \min \left\{\frac{m}{2}, \delta\right\} \cdot\|\mathbb{U}\|_{\mathbb{Y}}^{2} .
$$

On the other hand, from the condition of theorem it follows that

$$
\exists M_{1}>0: \quad|a(\mathbb{U}, \mathbb{V})| \leq M_{1} \cdot\|\mathbb{U}\|_{\mathbb{Y}} \cdot\|\mathbb{V}\|_{\mathbb{Y}} \quad \forall \mathbb{U}, \mathbb{V} \in \mathbb{Y} .
$$

Therefore, when $V$ and $E$ are complex Hilbert spaces, by Theorem 3.6.1 in [26] the operator $\mathbb{A}-\omega I$ and its restriction generate analytic semigroups, respectively, on the space $V^{n-1} \times V^{*}$ and $V^{n-1} \times E$. Thus, in the case of complex spaces the theorem is proved, because the value of $\omega$ is no mater. (cf. Remark 3.3 .2 of ch. 3 in [26]) When $V$ and $E$ are real Hilbert spaces, the theorem is proved by complexification. $\square$

REMARK 4.1. For the pseudo-hyperbolic systems, the condition in Theorem 4.1 is more useful than one in [5], because it is not required that $A_{i}$ are self-adjoint and positive-definite.

Let us study an initial value problem

$$
u^{(n)}+A u^{(n-1)}+f\left(t, u, \cdots, u^{(n-1)}\right)=0,
$$




$$
u(0)=u_{0}, u^{\prime}(0)=u_{0}^{\prime}, \cdots, u^{(n-1)}(0)=u_{0}^{(n-1)},
$$

where $V$ and $E$ are Hilbert spaces such that $V \hookrightarrow E$. Let $V_{\theta}^{*}=\left[V, V^{*}\right]_{1-\theta}, 0 \leq \theta \leq 1$ (complex interpolation space, see [17]).

Lemma 4.2. Suppose that the conditions of Theorem 4.1 are satisfied and $\mathscr{D}\left((-\mathbb{A}+\omega I)^{\theta}\right), 0<\theta \leq 1$, is the Banach space with a norm equivalent to the graph norm of $(-\mathbb{A}+\omega I)^{\theta}$, where $\omega$ is the number in (4.1).

Then, the space $\mathscr{D}\left((-\mathbb{A}+\omega I)^{\theta}\right)$ is continuously imbedded into the space $V^{n-1} \times V_{\theta^{\prime}}^{*}$, $0 \leq \theta^{\prime} \leq \theta \leq 1$.

Proof. Let $\mathbb{Y}=V^{n}, \mathbb{Y}^{*}=V^{n-1} \times V^{*}$ and $J \in\left(\mathbb{Y} \rightarrow \mathbb{Y}^{*}\right)$ be the duality operator. Then, the interpolation space $\left[\mathbb{Y}, \mathbb{Y}^{*}\right]_{1-\theta}$ is the space $\mathscr{D}\left(J^{\theta}\right)$ with a norm equivalent to $\left\|J^{\theta} \mathbb{U}\right\|_{\mathbb{Y}^{*}}$ and $\left[\mathbb{Y}, \mathbb{Y}^{*}\right]_{1-\theta}=V^{n-1} \times\left[V, V^{*}\right]_{1-\theta}$. By proposition 2.3, ch. 1 in [17],

$$
\|\mathbb{U}\|_{\mathscr{D}\left(J^{\theta}\right)} \leq C\|\mathbb{U}\|_{\mathbb{Y}}^{\theta} \cdot\|\mathbb{U}\|_{\mathbb{Y}^{*}}^{1-\theta} \quad \forall \mathbb{U} \in \mathbb{Y}
$$

On the other hand, by (4.2) we have

$$
\exists \omega_{1}>0:\|(-\mathbb{A}+\omega I) \mathbb{U}\|_{\mathbb{Y}^{*}} \geq \omega_{1}\|\mathbb{U}\|_{\mathbb{Y}}
$$

By virtue of (4.5) and (4.6), we get

$$
\|\mathbb{U}\|_{\mathscr{D}\left(J^{\theta}\right)} \leq C_{1}\|(-\mathbb{A}+\omega I) \mathbb{U}\|_{\mathbb{Y}^{*}}^{\theta} \cdot\|\mathbb{U}\|_{\mathbb{Y}^{*}}^{1-\theta} \quad \forall \mathbb{U} \in \mathbb{Y} .
$$

By Theorem 4.1, the operator $\mathbb{A}-\omega I$ is a generator of an analytic semigroup in $\mathbb{Y}^{*}$, and so $-\mathbb{A}+\omega I$ is sectorial. Thus, by (4.7) (cf. Exercise 11, section 4, ch.1, in [11])

$$
\left\|J^{\theta} \mathbb{U}\right\|_{\mathbb{Y}^{*}} \leq C\|\mathbb{U}\|_{\mathscr{D}\left((-\mathbb{A}+\omega I)^{\theta}\right)} \quad \forall \mathbb{U} \in \mathscr{D}\left((-\mathbb{A}+\omega I)^{\theta}\right)
$$

which means

$\mathscr{D}\left((-\mathbb{A}+\omega I)^{\theta}\right) \hookrightarrow \mathscr{D}\left(J^{\theta}\right)=V^{n-1} \times\left[V, V^{*}\right]_{1-\theta} \hookrightarrow V^{n-1} \times\left[V, V^{*}\right]_{1-\theta^{\prime}}=V^{n-1} \times V_{\theta^{\prime}}^{*}$

THEOREM 4.3. Suppose the following conditions are satisfied:

1) $A \in \mathcal{B L}\left(V, V^{*}\right)$ and there exists $m>0$ and a real number $k$ such that

$$
R e\langle A u, u\rangle_{V} \geq m\|u\|_{V}^{2}+k\|u\|_{E}^{2} \quad \forall u \in V ;
$$

2) $f \in\left([0, T] \times V^{n-1} \times V_{\theta}^{*} \rightarrow V^{*}\right)$ and $\forall t, \tau \in[0, T], \forall r>0, \forall \mathbb{U}, \mathbb{V} \in \mathscr{O}_{r}:$

$$
\|f(t, \mathbb{U})-f(\tau, \mathbb{V})\|_{V^{*}} \leq K(r)\left(|t-\tau|^{\varepsilon}+\|\mathbb{U}-\mathbb{V}\|_{V^{n-1} \times V_{\theta}^{*}}\right), 0 \leq \varepsilon \leq 1,0 \leq \theta<1,
$$

where $\mathscr{O}_{r}$ is the $r$-neighborhood of zero element of $V^{n-1} \times V_{\theta}^{*}$;

3) $\mathbb{U}_{0} \equiv\left(u_{0}, u_{0}^{\prime}, \cdots, u_{0}^{(n-1)}\right) \in V^{n-1} \times V_{\theta}^{*}$.

Then, the initial value problem (4.3), (4.4) has a unique local solution $u \in C^{n-2}\left(\left[0, t_{0}\right) ; V\right) \cap C^{n-1}\left(\left(0, t_{0}\right) ; V\right) \cap C^{n-1}\left(\left[0, t_{0}\right) ; V^{*}\right) \cap C^{n}\left(\left(0, t_{0}\right) ; V^{*}\right)$, 
where $t_{0}>0$.

If, in addition, $K(r)$ is independent of $r$ and $T=\infty$, then there exists a unique solution on $[0, \infty)$.

Proof. The first conclusion is equivalent to the existence of a unique solution $\mathbb{U} \in C\left(\left[0, t_{0}\right)\right.$ $\left.V^{n-1} \times V^{*}\right) \cap C^{1}\left(\left(0, t_{0}\right) ; V^{n-1} \times V^{*}\right)$ to problem

$$
\begin{aligned}
& \dot{\mathbb{U}}-(\mathbb{A}-\omega I) \mathbb{U}=F(t, \mathbb{U}), \\
& \mathbb{U}(0)=\mathbb{U}_{0},
\end{aligned}
$$

where

$$
\mathbb{A}=\left(\begin{array}{ccccc}
0 & 1 & 0 & \cdots & 0 \\
0 & 0 & 1 & \cdots & 0 \\
& \cdots & & \cdots & \\
0 & 0 & 0 & \cdots & -A
\end{array}\right), \quad F(t, \mathbb{U})=\left(\begin{array}{c}
\omega u \\
\omega u_{1} \\
\vdots \\
-f(t, \mathbb{U})+\omega u_{n-1}
\end{array}\right)
$$

and $\omega$ is the number in (4.1) with $A_{i}=0(i=0 \sim n-1), A_{n-1}=A$. From the condition 2) of theorem, we have

$$
\begin{aligned}
&\|F(t, \mathbb{U})-F(\tau, \mathbb{V})\|_{\mathbb{Y}^{*}} \leq\left\{\omega^{2} \sum_{i=0}^{n-2}\left\|u_{i}-v_{i}\right\|_{V}^{2}\right. \\
&+\left[K ( r ) \left(|t-\tau|^{\varepsilon}+\right.\right.\left.\left.\left.\|\mathbb{U}-\mathbb{V}\|_{V^{(n-1)} \times V_{\theta}^{*}}\right)+\omega\left\|u_{n-1}-v_{n-1}\right\|_{V^{*}}+K|t-\tau|^{\varepsilon}\right]^{2}\right\}^{\frac{1}{2}} \\
& \leq K^{\prime}(r)\left[|t-z|^{\varepsilon}+\|\mathbb{U}-\mathbb{V}\|_{V^{n-1} \times V_{\theta}^{*}}\right] \\
& \forall t, \tau \in[0, T], \quad \forall \mathbb{U}, \mathbb{V} \in \mathscr{O}_{r} .
\end{aligned}
$$

From (4.2) it follows that if $0 \leq \theta \leq \theta^{\prime}<1$, then the graph norm of $\mathbb{U}$ in $\mathscr{D}((-\mathbb{A}+$ $\left.\omega I)^{\theta^{\prime}}\right)$ is equivalent to $\left\|(-\mathbb{A}+\omega I)^{\bar{\theta}^{\prime}} \mathbb{U}\right\|_{\mathbb{Y}^{*}}$. And by Lemma $4.2, \mathscr{D}\left((-\mathbb{A}+\omega I)^{\theta^{\prime}}\right)$ is continuously embedded into $V^{(n-1)} \times V_{\theta^{\prime}}^{*}$. Thus, we have

$$
\begin{aligned}
\|\mathbb{U}\|_{V^{n-1} \times V_{\theta}^{*}} \leq K_{1}\|U\|_{\mathscr{D}\left((-\mathbb{A}+\omega I)^{\theta^{\prime}}\right)} & \left.\leq K_{2} \|(-\mathbb{A}+\omega I)^{\theta^{\prime}}\right) \mathbb{U} \|_{\mathbb{Y}^{*}} \\
& \forall \mathbb{U} \in \mathscr{D}\left((-\mathbb{A}+\omega I)^{\theta^{\prime}}\right),
\end{aligned}
$$

from which it follows that if $\left\|(-\mathbb{A}+\omega I)^{\theta^{\prime}} \mathbb{U}\right\|_{\mathbb{Y}^{*}},\left\|(-\mathbb{A}+\omega I)^{\theta^{\prime} \mathbb{V}}\right\|_{\mathbb{Y}^{*}}<r_{1} \equiv \frac{r}{K_{2}}$, then $\mathbb{U}, \mathbb{V} \in \mathscr{O}_{r}$. Therefore, from (4.9) we have that if $t, \tau \in[0, T], \mathbb{U}, \mathbb{V} \in \mathscr{D}\left((-\mathbb{A}+\omega I)^{\theta^{\prime}}\right)$ and $\left\|(-\mathbb{A}+\omega I)^{\theta^{\prime}} \mathbb{U}\right\|_{\mathbb{Y}^{*}},\left\|(-\mathbb{A}+\omega I)^{\theta^{\prime} \mathbb{V}}\right\|_{\mathbb{Y}^{*}}<r_{1}$, then

$$
\|F(t, \mathbb{U})-F(\tau, \mathbb{V})\|_{\mathbb{Y}^{*}} \leq K^{\prime \prime}(r)\left(|t-\tau|^{\varepsilon}+\|\mathbb{U}-\mathbb{V}\|_{\mathscr{D}\left((-\mathbb{A}+\omega I)^{\theta^{\prime}}\right)}\right)
$$

Consequently, by Theorem 3.1 of ch. 6 in [21], there exists a unique local solution to problem (4.8).

Let us prove the second conclusion.

By (4.2) the number 0 belongs to the resolvent set $\rho((\mathbb{A}-\omega I))$ and the analytic semigroup $T(t)$ generated by the operator $\mathbb{A}-\omega I$ is bounded on $[0, \infty)$. And if $t \in[0, \infty)$ and $\mathbb{U} \in \mathscr{D}\left((-\mathbb{A}+\omega I)^{\theta^{\prime}}\right), 0 \leq \theta<\theta^{\prime}<1$, then

$$
\begin{aligned}
\|F(t, \mathbb{U})\|_{\mathbb{Y}^{*}} & \leq\left\|F(t, \mathbb{U})-F\left(0,0_{\mathbb{Y}}\right)\right\|_{\mathbb{Y}^{*}}+\left\|F\left(0,0_{\mathbb{Y}}\right)\right\|_{\mathbb{Y}^{*}} \\
& \leq K\left(t^{\varepsilon}+\|\mathbb{U}\|_{V^{n-1} \times V_{\theta}^{*}}\right)+\left\|F\left(0,0_{\mathbb{Y}}\right)\right\|_{\mathbb{Y}^{*}}
\end{aligned}
$$


Define

$$
k(t)= \begin{cases}K+\left\|F\left(0,0_{\mathbb{Y}}\right)\right\|_{\mathbb{Y}^{*}} & t \in[0,1] \\ K t^{\varepsilon}+\left\|F\left(0,0_{\mathbb{Y}}\right)\right\|_{\mathbb{Y}^{*}} & t>1,\end{cases}
$$

which is a continuous nondecreasing function. Then, taking into account Lemma 4.2, we have

$$
\|F(t, \mathbb{U})\|_{\mathbb{Y}^{*}} \leq k(t)\left(1+\|\mathbb{U}\|_{V^{n-1} \times V_{\theta}^{*}}\right) \leq K k(t)\left(1+\|\mathbb{U}\|_{\mathscr{D}\left((-\mathbb{A}+\omega I)^{\theta^{\prime}}\right)}\right) .
$$

Thus, by Theorem 3.3 of ch. 6 in [21], we come to the second conclusion. $\square$

Let us study another initial value problem

$$
\begin{aligned}
u^{(n)} & +A_{n-1}\left(t, u, \cdots, u^{(n-1)}\right) u^{(n-1)} \\
& +\cdots+A_{0}\left(t, u, \cdots, u^{(n-1)}\right) u=f\left(t, u, \cdots, u^{(n-1)}\right),
\end{aligned}
$$

where $A_{i}\left(t, v, \cdots, v_{n-1}\right) \in \mathcal{B} \mathcal{L}\left(V, V^{*}\right)$ for every fixed $\left(t, v, \cdots, v_{n-1}\right) \quad \in$ $[0, T] \times V^{n-1} \times V_{\theta}^{*}, f \in\left([0, T] \times V^{n-1} \times V_{\theta}^{*} \rightarrow V^{*}\right)$ and $0 \leq \theta<1$.

First, we study an equation

$$
u^{(n)}+A_{n-1}\left(t, u, \cdots, u^{(n-1)}\right) u^{(n-1)}=f\left(t, u, \cdots, u^{(n-1)}\right) .
$$

THEOREM 4.4. Suppose that

1) There exists $m>0$ and a real number $k$ such that

$$
R e\left\langle A_{n-1}\left(0, \mathbb{U}_{0}\right) u, u\right\rangle_{V} \geq m\|u\|_{V}^{2}+k\|u\|_{E}^{2} \quad \forall u \in V
$$

2) For $\mathbb{U}_{1}, \mathbb{U}_{2} \in \mathscr{O}_{r}$ and $t, \tau \in[0, T]$,

$$
\left\|A_{n-1}\left(t, \mathbb{U}_{1}\right)-A_{n-1}\left(\tau, \mathbb{U}_{2}\right)\right\|_{V \rightarrow V^{*}} \leq K(r)\left(|t-\tau|^{\varepsilon}+\left\|\mathbb{U}_{1}-\mathbb{U}_{2}\right\|_{V^{n-1} \times V_{\theta}^{*}}\right)
$$

and

$\left\|f\left(t, \mathbb{U}_{1}\right)-f\left(\tau, \mathbb{U}_{2}\right)\right\|_{V^{*}} \leq K(r)\left(|t-\tau|^{\varepsilon}+\left\|\mathbb{U}_{1}-\mathbb{U}_{2}\right\|_{V^{n-1} \times V_{\theta}^{*}}\right)$,

where $0 \leq \varepsilon \leq 1, \mathscr{O}_{r}$ is the same as in Theorem 4.2 and $\|\cdot\|_{V \rightarrow V^{*}}$ means the norm of the space $\mathcal{B} \mathcal{L}\left(V, V^{*}\right)$,

3) $\mathbb{U}_{0} \equiv\left(u_{0}, u_{0}^{\prime}, \cdots, u_{0}^{(n-1)}\right) \in V^{n}$.

Then, the initial value problem (4.11),(4.12) has a unique local solution on $\left[0, t_{0}\right), t_{0}>0$.

Proof. The conclusion is equivalent to the existence of a unique solution

$$
\mathbb{U} \in C\left(\left[0, t_{0}\right) ; V^{n-1} \times V^{*}\right) \cap C^{1}\left(\left(0, t_{0}\right) ; V^{n-1} \times V^{*}\right)
$$

to problem

$$
\begin{aligned}
& \dot{U}-\mathbb{A}_{1}(t, \mathbb{U}) \mathbb{U}=F_{1}(t, \mathbb{U}), \\
& \mathbb{U}(0)=\mathbb{U}_{0},
\end{aligned}
$$


where

$$
\mathbb{A}_{1}(t, \mathbb{U})=\left(\begin{array}{cccc}
0 & 1 & \cdots & 0 \\
& \cdots & & \cdots \\
0 & 0 & \cdots & 1 \\
0 & 0 & \cdots & -A_{n-1}(t, \mathbb{U})
\end{array}\right)-\omega I, \quad F_{1}(t, \mathbb{U})=\left(\begin{array}{c}
\omega u \\
\omega u_{1} \\
\vdots \\
f(t, \mathbb{U})+\omega u_{n-1}
\end{array}\right),
$$

and $\omega$ is the number in (4.8) with $A=A_{n-1}\left(0, \mathbb{U}_{0}\right)$.

Using (3.51) of Lemma 3.6.1 in [26], we obtain

$$
\left\|\left[\mathbb{A}_{1}\left(0, \mathbb{U}_{0}\right)+\lambda\right]^{-1}\right\|_{\mathbb{Y}^{*} \rightarrow \mathbb{Y}^{*}} \leq \frac{M_{1}}{|\lambda|} \quad \text { for } \operatorname{Re} \lambda \geq 0 .
$$

On the other hand, from (4.2) it follows that there exists $\delta_{1}>0$ such that

$$
\left\|\mathbb{A}_{1}\left(0, \mathbb{U}_{0}\right)^{-1} \mathbb{U}\right\|_{\mathbb{Y}} \leq \delta_{1}\|\mathbb{U}\|_{\mathbb{Y}^{*}} \forall \mathbb{U} \in \mathbb{Y}^{*}
$$

where $\mathbb{Y}^{*}$ is the same as in Theorem 4.1. Inequality (4.14) implies that the number 0 belongs to the resolvent set $\rho\left(\mathbb{A}_{1}\left(0, \mathbb{U}_{0}\right)\right)$. This fact together with (4.13) implies that

$$
\left\|\left[\mathbb{A}_{1}\left(0, \mathbb{U}_{0}\right)+\lambda\right]^{-1}\right\|_{\mathbb{Y}^{*} \rightarrow \mathbb{Y}^{*}} \leq \frac{M_{2}}{1+|\lambda|} \quad \text { for } \operatorname{Re} \lambda \geq 0 .
$$

In view of (4.14) and the condition 2) of theorem, we know that

$$
\begin{aligned}
& \|\left[\mathbb{A}_{1}\left(t, \mathbb{U}_{1}\right)-\mathbb{A}_{1}\left(\tau, \mathbb{U}_{2}\right)\right] \cdot \mathbb{A}_{1}\left(0, \mathbb{U}_{0}\right)^{-1} \|_{\mathbb{Y}^{*} \rightarrow \mathbb{Y}^{*}} \\
& \leq\left\|A_{n-1}\left(t, \mathbb{U}_{1}\right)-A_{n-1}\left(\tau, \mathbb{U}_{2}\right)\right\|_{V \rightarrow V^{*}} \cdot \delta_{1} \\
& \leq K^{\prime}(r)\left(|t-\tau|^{\varepsilon}+\left\|\mathbb{U}_{1}-\mathbb{U}_{2}\right\|_{V^{n-1} \times V_{\theta}^{*}}\right) \\
& \quad \forall t, \tau \in[0, T], \forall \mathbb{U}_{1}, \mathbb{U}_{2} \in \mathscr{O}_{r} .
\end{aligned}
$$

Moreover, as the proof of Theorem 4.3 we have that

$$
\begin{gathered}
\left\|F_{1}\left(t, \mathbb{U}_{1}\right)-F_{1}\left(\tau, \mathbb{U}_{2}\right)\right\|_{\mathbb{Y}^{*}} \leq K^{\prime}(r)\left(|t-\tau|^{\varepsilon}+\left\|\mathbb{U}_{1}-\mathbb{U}_{2}\right\|_{V^{n-1} \times V_{\theta}^{*}}\right) \\
\forall t, \tau \in[0, T], \forall \mathbb{U}_{1}, \mathbb{U}_{2} \in \mathscr{O}_{r} .
\end{gathered}
$$

Let us take $\theta^{\prime}$ such that $0 \leq \theta<\theta^{\prime}<1$. Since $\mathbb{U}_{0} \in \mathbb{Y}$ and $\mathbb{A}_{1}\left(0, \mathbb{U}_{0}\right) \in \mathcal{B} \mathcal{L}\left(\mathbb{Y}, \mathbb{Y}^{*}\right)$, there exists a number $r_{1}>0$ such that

$$
\left\|\mathbb{A}_{1}^{\theta^{\prime}}\left(0, \mathbb{U}_{0}\right) \cdot \mathbb{U}_{0}\right\|_{\mathbb{Y}^{*}} \leq K\left\|\mathbb{A}_{1}\left(0, \mathbb{U}_{0}\right) \cdot \mathbb{U}_{0}\right\|_{\mathbb{Y}^{*}}<r_{1} .
$$

On the other hand, by Lemma 4.2 we have

$$
\begin{aligned}
\left\|\mathbb{A}_{1}^{-\theta^{\prime}}\left(0, \mathbb{U}_{0}\right) \cdot \mathbb{U}\right\|_{V^{n-1} \times V_{\theta}^{*}} & \leq K_{1}\left\|\mathbb{A}_{1}^{\theta^{\prime}}\left(0, \mathbb{U}_{0}\right) \cdot \mathbb{A}_{1}^{-\theta^{\prime}}\left(0, \mathbb{U}_{0}\right) \cdot \mathbb{U}\right\|_{\mathbb{Y}^{*}} \\
& \leq K_{1}\|\mathbb{U}\|_{\mathbb{Y}^{*}} \quad \forall \mathbb{U} \in \mathbb{Y}^{*} .
\end{aligned}
$$

Thus, if $\|\mathbb{U}\|_{\mathbb{Y}^{*}} \leq r_{2} \equiv \frac{r}{K_{1}}$, then $\mathbb{A}_{1}^{-\theta^{\prime}}\left(0, \mathbb{U}_{0}\right) \mathbb{U}$ belongs to $\mathscr{O}_{r}$. Therefore, substituting $\mathbb{U}_{i}$ with $\mathbb{A}_{1}^{-\theta^{\prime}}\left(0, \mathbb{U}_{0}\right) \mathbb{U}_{i}$ in $(4.15),(4.16)$ and applying Lemma 4.2 , we have that if $\left\|\mathbb{U}_{1}\right\|_{\mathbb{Y}^{*}},\left\|\mathbb{U}_{2}\right\|_{\mathbb{Y}^{*}} \leq r_{2}$, then

$$
\begin{aligned}
\left\|\left[\mathbb{A}_{1}\left(t, \mathbb{A}_{1}^{-\theta^{\prime}}\left(0, \mathbb{U}_{0}\right) \mathbb{U}_{1}\right)-\mathbb{A}_{1}\left(\tau, \mathbb{A}_{1}^{-\theta^{\prime}}\left(0, \mathbb{U}_{0}\right) \mathbb{U}_{2}\right)\right] \mathbb{A}_{1}\left(0, \mathbb{U}_{0}\right)^{-1}\right\|_{\mathbb{Y}^{*} \rightarrow \mathbb{Y}^{*}} \\
\leq K_{2}(r)\left(|t-\tau|^{\varepsilon}+\left\|\mathbb{U}_{1}-\mathbb{U}_{2}\right\|_{\mathbb{Y}^{*}}\right), \\
\left\|F_{1}\left(t, \mathbb{A}_{1}^{-\theta^{\prime}}\left(0, \mathbb{U}_{0}\right) \mathbb{U}_{1}\right)-F_{1}\left(\tau, \mathbb{A}_{1}^{-\theta^{\prime}}\left(0, \mathbb{U}_{0}\right) \mathbb{U}_{2}\right)\right\|_{\mathbb{Y}^{*}} \\
\leq K_{3}(r)\left(|t-\tau|^{\varepsilon}+\left\|\mathbb{U}_{1}-\mathbb{U}_{2}\right\|_{\mathbb{Y}^{*}}\right) .
\end{aligned}
$$


Consequently, by Theorem 7 of [24], from (4.17), (4.18) we get the conclusion.

Corollary 4.5. Assume that the conditions in Theorem 4.4 are satisfied. If condition 2) for $A_{n-1}$ is valid for $A_{i}, i=0,1, \cdots, n-2$, then problem (4.10), (4.11) has a unique local solution on $\left[0, t_{0}\right), t_{0}>0$.

Proof. When $A_{i}\left(t, u, \cdots, u^{(n-1)}\right) u^{(i)}, i=0,1, \cdots, n-2$, are included into $f$, problem (4.10), (4.11) is reduced to problem (4.11), (4.12). Thus, Theorem 4.4 implies the corollary.

REMARK 4.2. If condition 1) in Theorem 4.4 is satisfied for any $\mathbb{U}_{0} \in V^{n}$ and $K(r)$ is independent of $r$, we obtain existence of a global solution.

Applying the abstract results above, we can get unique existence of the solutions to systems of pseudo-hyperbolic equations, which are given below in Theorems 4.6 and 4.7 .

Let $\Omega$ be a bounded domain of $R^{n}$ of class $C^{\infty}$ and $\alpha=\left(\alpha_{1}, \cdots, \alpha_{n}\right)$, where $\alpha_{i}$

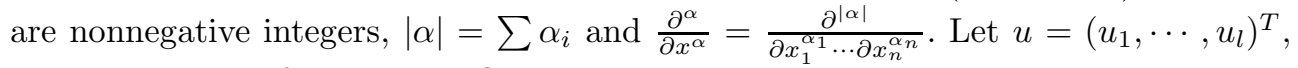
where $u_{i}$ are real functions on $\Omega$.

First, let us consider the following mixed problem of a system of pseudo-hyperbolic equations

$$
\begin{gathered}
u_{t t}+(-1)^{m} \sum_{|\alpha|=m} \frac{\partial^{\alpha}}{\partial x^{\alpha}}\left[A_{\alpha}(x) \frac{\partial^{\alpha}}{\partial x^{\alpha}} u_{t}\right]+\sum_{|\beta| \leq m} \frac{\partial^{\beta}}{\partial x^{\beta}} A_{\beta}^{\prime}\left(t, x, u_{t}, \cdots, \frac{\partial^{\gamma}}{\partial x^{\gamma}} u_{t}, \cdots\right) \\
+\sum_{|\alpha| \leq m} \frac{\partial^{\alpha}}{\partial x^{\alpha}} B_{\alpha}\left(t, x, u, \cdots, \frac{\partial^{\delta}}{\partial x^{\delta}} u, \cdots, u_{t}, \cdots, \frac{\partial^{\sigma}}{\partial x^{\sigma}} u_{t}, \cdots\right)=\sum_{|\alpha| \leq m} \frac{\partial^{\alpha}}{\partial x^{\alpha}} f_{\alpha}(t, x), \\
(4.20) \quad u(0, x)=u_{0}(x) \in H_{0}^{m}(\Omega)^{l}, \quad u_{t}(0, x)=u_{0}^{\prime}(x) \in H_{0}^{m-1}(\Omega)^{l}, \\
\left.\frac{\partial^{\alpha} u(t, x)}{\partial \nu^{\alpha}}\right|_{\partial \Omega}=0, \quad|\alpha| \leq m-1,
\end{gathered}
$$

where $|\gamma|,|\sigma| \leq m-1,|\delta| \leq m$, and $\nu$ is outward normal unit vector on $\partial \Omega$.

THEOREM 4.6. Suppose the following conditions hold:

1) Matrices $A_{\alpha}(x),|\alpha|=m$, are positive-definite at a.a. $x \in \Omega$ and their elements belong to $L_{\infty}(\Omega)$;

2) $A_{\beta}^{\prime} \in\left([0, \infty) \times \Omega \times R^{N} \rightarrow R^{l}\right)$ and

$\left\|A_{\beta}^{\prime}\left(t, x, \xi^{1}\right)-A_{\beta}^{\prime}\left(\tau, x, \xi^{2}\right)\right\|_{R^{l}} \leq K\left(|t-\tau|^{\varepsilon}+\sum_{i=1}^{N}\left|\xi_{i}^{1}-\xi_{i}^{2}\right|\right), 0 \leq \varepsilon \leq 1, \forall \xi_{1}, \xi_{2} \in R^{N}$

3) $B_{\alpha} \in\left([0, \infty) \times \Omega \times R^{N_{1}} \rightarrow R^{l}\right)$ and

$$
\left\|B_{\alpha}\left(t, x, \eta^{1}\right)-B_{\alpha}\left(\tau, x, \eta^{2}\right)\right\|_{R^{l}} \leq K_{1}\left(|t-\tau|^{\varepsilon}+\sum_{i=1}^{N_{1}}\left|\eta_{i}^{1}-\eta_{i}^{2}\right|\right) \forall \eta^{1}, \eta^{2} \in R^{N_{1}} ;
$$

4) $f_{\alpha}(t, \cdot) \in L_{2}(\Omega)^{l}$ and $\left\|f_{\alpha}(t, x)-f_{\alpha}(\tau, x)\right\|_{L_{2}(\Omega)^{l}} \leq K|t-\tau|^{\varepsilon}$. 
Then, problem $(4.19) \sim(4.21)$ has a unique solution

$$
\begin{aligned}
u(t, x) \in C\left([0, \infty) ; H_{0}^{m}(\Omega)^{l}\right) & \cap C^{1}\left((0, \infty) ; H_{0}^{m}(\Omega)^{l}\right) \\
& \cap C^{1}\left([0, \infty) ; H^{-m}(\Omega)^{l}\right) \cap C^{2}\left((0, \infty) ; H^{-m}(\Omega)^{l}\right) .
\end{aligned}
$$

Next, let us consider the following mixed problem

$$
\begin{aligned}
& u_{t t}+(-1)^{m} \sum_{|\alpha|=m} \frac{\partial^{\alpha}}{\partial x^{\alpha}}\left[A_{\alpha}\left(t, x, u, \cdots, \frac{\partial^{\beta} u}{\partial x^{\beta}}, \cdots, u_{t}, \cdots, \frac{\partial^{\gamma} u_{t}}{\partial x^{\gamma}}, \cdots\right) \frac{\partial^{\alpha} u_{t}}{\partial x^{\alpha}}\right] \\
& +\sum_{|\alpha| \leq m,|\delta| \leq m-1} \frac{\partial^{\alpha}}{\partial x^{\alpha}}\left[A_{\alpha \delta}\left(t, x, u, \cdots, \frac{\partial^{\beta} u}{\partial x^{\beta}}, \cdots, u_{t}, \cdots, \frac{\partial^{\gamma} u_{t}}{\partial x^{\gamma}}, \cdots\right) \frac{\partial^{\delta} u_{t}}{\partial x^{\delta}}\right] \\
& +\sum_{|\alpha| \leq m,|\rho| \leq m} \frac{\partial^{\alpha}}{\partial x^{\alpha}}\left[B_{\alpha \rho}\left(t, x, u, \cdots, \frac{\partial^{\beta} u}{\partial x^{\beta}}, \cdots, u_{t}, \cdots, \frac{\partial^{\gamma} u_{t}}{\partial x^{\gamma}}, \cdots\right) \frac{\partial^{\rho} u}{\partial x^{\rho}}\right] \\
& +\sum_{|\alpha| \leq m} \frac{\partial^{\alpha}}{\partial x^{\alpha}} F_{\alpha}\left(t, x, u, \cdots, \frac{\partial^{\sigma} u}{\partial x^{\sigma}}, \cdots, u_{t}, \cdots, \frac{\partial^{q} u_{t}}{\partial x^{q}}, \cdots\right) \\
& =\sum_{|\alpha| \leq m} \frac{\partial^{\alpha}}{\partial x^{\alpha}} G_{\alpha}(t, x), \\
& u(0, x)=u_{0}(x), u_{t}(0, x)=u_{0}^{\prime}(x) \in H_{0}^{m}(\Omega)^{l}, \\
& \frac{\partial^{\alpha} u(t, x)}{\partial \gamma^{\alpha}} \mid \begin{array}{l}
\partial \Omega=0, \quad|\alpha| \leq m-1,
\end{array}
\end{aligned}
$$

where $|\sigma| \leq m,|q| \leq m-1,|\beta|,|\gamma|<m-n / 2$.

THEOREM 4.7. Suppose the following conditions hold:

1) The matrices $A_{\alpha}\left(0, x, u_{0}(x), \cdots, \frac{\partial^{\beta} u_{0}(x)}{\partial x^{\beta}}, \cdots, u_{0}^{\prime}(x), \cdots, \frac{\partial^{\gamma} u_{0}^{\prime}(x)}{\partial x^{\gamma}}, \cdots\right)$ are positive definite at a.a. $x \in \Omega$;

2) As a function of $(t, x, \xi) \in[0, T] \times \Omega \times R^{N}$, every component $a(t, x, \xi)$ of $A_{\alpha}, A_{\alpha \delta}, B_{\alpha \rho}$ and $F_{\alpha}$ is bounded and measurable with respect to $x$ at every fixed $(t, \xi)$, and continuous with respect to $(t, \xi)$ at a.a. $x \in \Omega$;

3) $\left|a(t, x, \xi)-a\left(\tau, x, \xi^{1}\right)\right| \leq K(r)\left(|t-\tau|^{\varepsilon}+\sum_{n=1}^{N}\left|\xi_{i}-\xi_{i}^{1}\right|\right)$ for $t, \tau \in[0, T]$ and $\xi, \xi^{1} \in R^{N}\left(|\xi|_{R^{N}},\left|\xi^{1}\right|_{R^{N}}<r\right)$;

4) $G_{\alpha}(t, \cdot) \in L_{2}(\Omega)^{l}$ and $\left\|G_{\alpha}(t, x)-G_{\alpha}(\tau, x)\right\|_{L^{2}(\Omega)} \leq K|t-\tau|^{\varepsilon}$, where integer $N$ depends on $A_{\alpha}, A_{\alpha \delta}, B_{\alpha \rho}$ and $F$, and $0 \leq \varepsilon \leq 1$.

Then, there exists a unique local solution

$$
\begin{aligned}
u(t, x) \in C\left(\left[0, t_{0}\right) ; H_{0}^{m}(\Omega)^{l}\right) & \cap C^{1}\left(\left(0, t_{0}\right) ; H_{0}^{m}(\Omega)^{l}\right) \\
& \cap C^{1}\left(\left[0, t_{0}\right) ; H^{-m}(\Omega)^{l}\right) \cap C^{2}\left(\left(0, t_{0}\right) ; H^{-m}(\Omega)^{l}\right)
\end{aligned}
$$

to problem $(4.22) \sim(4.24)$.

REMARK 4.3. For equation (2) of [25], it was assumed that $B_{\alpha}$ are independent of $u_{t}$ and their derivatives with respect to $x, l=1, B_{\alpha}(|\alpha|=m)$ are linear and matrices $A_{\alpha}, B_{\alpha}(|\alpha|=m)$ are symmetric. For linear equation (7) of [25], it was assumed that $A_{\alpha}, B_{\alpha}(|\alpha|=m)$ are symmetric and $l=1$. 
Acknowledgments. The authors would like to thank to Prof. J. L. Lions and Yanpin Lin for their comments for our original preprints. We also thank Prof. G. O. H. Katona for his attention to our original preprints. The authors are grateful to the anonymous referee for his or her valuable comments.

\section{REFERENCES}

[1] N. C. Apreutesei, Nonlinear second order evolution equations of monotone type and applications, Pushpa Publishing House, Allahabad, 2007.

[2] H. Assa And M. HesaAraki, Nonexistence of solution for higher order evolution equations and inequalities, Methods and Applications of Analysis, 12:1 (2005), pp. 1-18.

[3] P. Aviles and J. Sandefur, Nonlinear second order equations with applications to partial differential equations, J. Diff. Equat., 58:3 (1985), pp. 404-427.

[4] G. Chen, H. Guo, and H. Zhang, Global existence of solutions of Cauchy problem for generalized system of nonlinear evolution equations arising from DNA, J. Math. Phys., 50, 083514 (2009), pp. 1-23.

[5] S. Chen and R. Triggiani, Proof of extension of two conjectures on stractural damping for elastic system, Pacific J. Math., 136:1 (1989), pp. 15-55.

[6] M. G. Crandall and T. M. LigGett, Generation of semi-groups of nonlinear transformations on general Banach spaces, American Journal of Mathematics, 93:2 (1971), pp. 265-298.

[7] H. Gajewski, K. Gröger, And K. Zacharias, Nichtlineare operatorgleichungen und operatordifferentialgleichungen, Akademie-Verlag, 1974 (Russian version, Mir, 1978).

[8] D. GuidetTi, On boundary value problems for parabolic equations of higher order in time, J. Diff. Equat., 124:1 (1996), pp. 1-26.

[9] K. Deimling, Nonlinear functional analysis, Springer-Verlag, 1985

[10] Yu. A. DubinskiI, On some differential-operator equations of arbitrary order, Math. USSR Sbornik, 19:1 (1973), pp. 1-21.

[11] D. Henry, Geometric theory of semilinear parabolic equations, Lecture Notes in Mathematics, 840, Springer-Verlag, 1981.

[12] N. D. Kopachevsky, R. Mennicken, Ju. S. Pashrova and C. Tretter, Complete second order linear differential operator equations in Hilbert space and applications in hydrodynamics, Trans. Amer. Math. Soc., 356 (2004), pp. 4737-4766.

[13] S. G. Krein, Linear differential equations in Banach spaces, AMS, Providence, 1971.

[14] S. G. Krein and M. I. Hazian, Differential equations in Banach spaces, Itogi Nauki i Tehniki, Mathematical Analyis, 21 (1983), pp. 130-263 (in Russian).

[15] L. I. Kuchminskaya, Nonlinear differential-operator equations and their applications, Dokl. Akad. Nauk USSR, 278:1 (1984), pp. 27-30.

[16] J. LiAng AND T. XIAO, Well-posedness results for certain classes of Higher order abstract Cauchy problems connected with integrated semigroups, Semigroup Forum, 56:1 (1998), pp. 84-103.

[17] J. L. Lions And E. Magenes, Non-homogeneous boundary value problems and applications, I, Springer-Verlag, 1972.

[18] M. Necula, M. Popescu, and I. I. Vrabie, Nonlinear evolution equations on locally closed graphs, RACSAM, 104:1 (2010), pp. 97-114.

[19] F. Neubrander, Well-posedness of higher order abstract Cauchy problems, Trans. American Math. Society, 295:1 (1986), pp. 257-290.

[20] Е. ОвRеснт, The Cauchy problem for time-dependent abstract parabolic equations of higher order, J. Math. Anal. Appl., 125:2 (1987), pp. 508-530.

[21] A. PAZY, Semigroups of linear operators and applications to partial differential equations, Springer-Verlag, 1983.

[22] J. SAndefur, Higher order abstract Cauchy problem, J. Math. Anal. Appl., 60:3 (1977), pp. $728-742$.

[23] J. SANDEFUR, Existence and uniqueness of solutions of second order nonlinear differential equations, SIAM J. Math. Anal., 14:3 (1983), pp. 477-487.

[24] P. E. Sobolevskil, Equations of parabolic type in a Banach space, Amer. Math. Soc. Transl, 49:2 (1965), pp. 1-62.

[25] H. Sun, A nonlinear pseudo-hyperbolic system, Science in China, A, 32:1 (1989), pp. 1-19.

[26] H. TANABE, Equations of evolution, Pitman, 1979.

[27] T. XiaO And J. Liang, Entire solutions of higher order abstract Cauchy problems, J. Math. Anal. Appl., 208:2 (1997), pp. 298-310. 
[28] T. XIAO AND J. LIANG, Exponential stability of solutions for higher order abstract Cauchy problems, Ibid., 215:2 (1997), pp. 485-498.

[29] T. XIAO And J. LiAng, The Cauchy problem for higher-order abstract differential equations, Lecture Notes in Mathematics 1701, Springer, 1998. 
T. KIM, Q. CHANG AND J. XU 